This manuscript is a preprint and has been submitted for publication in Geochemistry, Geophysics, Geosystems. Please note that, despite having undergone peer-review, the manuscript has yet to be formally accepted for publication. Subsequent versions of this manuscript may have slightly different content. If accepted, the final version of this manuscript will be available via the 'Peer-reviewed Publication DOl' link on the right-hand side of this webpage. Please feel free to contact any of the authors; we welcome feedback. 


\title{
The spectrum of slip behaviours of a granular fault gouge analogue governed by rate and state friction.
}

\author{
M. Rudolf ${ }^{1}$, M. Rosenau ${ }^{1}$, and O. Oncken ${ }^{1}$ \\ ${ }^{1}$ Helmholtz Centre Potsdam German Research Centre for Geosciences - GFZ, Telegrafenberg, 14473 \\ Potsdam, Germany
}

\section{Key Points:}

- Slip modes in granular gouge are akin to natural fault slip.

- Glass beads are a suitable granular analogue for fault gouge and show rate-andstate dependent friction.

- Enhanced creep and small scale events are signals for imminent failure and indicate fault criticality.

Corresponding author: M. Rudolf, michael.rudolf@gfz-potsdam.de 


\begin{abstract}
The exact principles of earthquake recurrence and magnitude are currently unknown which is why earthquake hazard assessment relies on statistical models combined with numerical simulations. A component of seismic and aseismic slip is the frictional character of a fault. We shear fused glass beads with a narrow particle size distribution of 300-400um at stresses of $5-20 \mathrm{kPa}$ and with low shear rates of less than $1 \mathrm{~mm} / \mathrm{s}$. As a result, we show that characteristic slip events emerge, ranging from fast and large slip to small scale oscillating creep and stable sliding. In particular we observe small scale slip events that occur immediately before large scale slip events for a specific set of experiments. Similar to natural faults we find a separation of scales by several orders of magnitude for slow events and fast events. Enhanced creep and transient dilatational events pinpoint that the granular analogue is close to failure. From slide-hold-slide tests, we find that the rateand-state properties are in the same range as estimates for natural faults and fault rocks. The fault shows velocity weakening characteristics with a reduction of frictional strength between 0.8 to $1.3 \%$ per e-fold increase in sliding velocity. Furthermore, the slip modes that are observed in the normal shear experiments are in good agreement with analytical solutions. Our findings highlight the influence of micromechanical processes on macroscopic fault behaviour. The comprehensive dataset associated with this study can act as a benchmark for numerical simulations and alleviate the understanding of observations of natural faults.
\end{abstract}

\title{
Plain Language Summary
}

Earthquakes occur when two continental plates slide along each other. The motion is concentrated at the interface of the two plates which is called a fault. In many cases the fault is filled with granular material, called gouge, that supports the pressure between the plates. Therefore, the properties of this gouge determine how fast and how large an earthquake can be. It also has an influence on the time between earthquakes. In our study we examine a simplified version of a fault gouge in a simple small-scale model. Instead of rock material we use glass beads and measure how different conditions affect the motion of the model. We find that our model reproduces features of fault gouge because it shows similar behaviour. When there is no motion our model fault becomes stronger with a rate equal to fault gouge. Also, the type of strengthening is analogous to fault gouge. During slip, the glass beads become weaker as the slip velocity increases in a similar manner as natural faults. These results improve the understanding of computer simulations and natural observations.

\section{Introduction}

Seismically active faults pose a major threat to many communities world-wide. Therefore, it is vital to make appropriate predictions on the probability of large earthquakes and their associated effects, such as tsunamis and mass movements. Several factors contribute to the difficulties to estimate seismic hazard in the vicinity of such faults. Besides the vulnerability of structures and the societal impact, geological factors play an important role in seismic hazard assessment and the development of models that describe fault activity (Zöller \& Hainzl, 2007). Current models for earthquake recurrence incorporate mathematical models of earthquake statistics (Gutenberg-Richter, Omori-UtsuAftershocks, Brownian-First-Passage-Time), numerical models of earthquakes and rupture processes (Rate-and-State-Friction), interseismic stress built-up and the interaction of multiple faults over a larger area via stress transfer (e.g. Brinkman et al., 2016; Ellsworth et al., 1999; Field et al., 2014; Hainzl et al., 2013; Hu \& Bradley, 2018; Kawamura et al., 2012; Lapusta \& Rice, 2003; Parsons, 2005; Zöller et al., 2011). These models inherently rely on the accurate description and characterization of fault properties and behaviour, as well as extensive catalogues of slip events. With this study we aim to characterize a 
physical scale model of seismic activity to expand models of seismic hazard assessment with experimental data and also show the potential impact of various slip modes on seismic activity.

\subsection{Fault Slip}

Active faults are characterized by a wide range of slip behaviours ranging from aseismic creep to seismic stick-slip that may change spatially along the fault and temporally over the seismic cycle (e.g. Harris, 2017; Peng \& Gomberg, 2010). The types of slip are defined by their characteristic timescale which ranges from milliseconds to a few years (Obara \& Kato, 2016) and by their characteristic magnitude which is usually defined by seismic moment (Ide et al., 2007; Gomberg et al., 2016). Depending on their characteristics in time and seismic wave forms, the slip events are characterized as seismic (very low frequency earthquakes, tremors, normal earthquake) or geodetic (short-term and longterm slow slip events) events. They can occur simultaneously, i.e. within one seismic cycle, at the same locality or in different depth ranges of the same main fault (Bürgmann, 2018). The physical origin of this range of slip modes is still not entirely clear, although several approaches for certain phenomena have been proposed (Daniels \& Hayman, 2008; Ciamarra et al., 2010; Chen \& Spiers, 2016; Dorostkar \& Carmeliet, 2018).

A common methodology to model this wide range of slip behaviours is through a continuum based description that reproduces the kinematics and dynamics of fault activity. The rate-and-state framework provides the possibility to characterize fault behaviour, or in a general term 'fault rheology', by describing the connection of forces in the system (friction $\mu$ ) and the external influences such as loading rate $v_{L}$ and stiffness $k$ (Brace \& Byerlee, 1966; Dieterich, 1978; J. H. Dieterich, 1979a, 1979b; Scholz, 1998). In general, the rate-and-state framework is able to describe most observations that lead to fault (in-)stability and has been derived from experimental observations in the laboratory and a few field observations (Marone, 1998, and references therein). Stick-slip experiments using rock and rock analogues suggest that besides intrinsic material properties (e.g. friction coefficient, slip/velocity weakening), extrinsic parameters like stiffness, normalized loading rate and effective normal stress are key controls of frictional stability (e.g. Leeman et al., 2016; Heslot et al., 1994; Marone, 1998; Mair et al., 2002). Recent studies also highlight that several of the fault intrinsic parameters in the rate-and-state equation are also dependent on extrinsic parameters and not constants as previously assumed (Van den Ende et al., 2018; Chen \& Spiers, 2016).

\subsection{Granular Fault Analogues}

In this study we purely focus on the frictional characteristics of an analogue fault zone which is described with the rate-and-state framework (J. H. Dieterich, 1979a; J. Dieterich, 2007). Our fault zone is composed of a granular fault core with relatively stiff outer boundaries and dominated by granular mechanics. Other processes that influence the slip modes along a fault zone, which are not realized in our setup, are variations in pore-fluid pressure, changes in material because of comminution, or mineral reactions. Not all slip modes are observed for all active zones which strongly suggests that there is a complex interaction between the processes acting on different scales in space and time. Knowledge of the complex interactions between the different slip modes is relevant for estimating the seismicity rates along plate boundaries and therefore for seismic hazard assessment. Other possible areas of application include soil mechanics and mass movements.

The advantage of using a granular analogue is the simplicity with which observations can be made. The analogue modelling approach features lower stresses which simplifies the design and construction of the testing machine. This increases the available parameter space because it is relatively easy to change the system stiffness using springs. 
For rock mechanical testing apparatuses the change in stiffness is limited to a smaller range that is either accessible through adding rubber blocks or by artificially changing the servo-hydraulic systems to mimic a different stiffness (Beeler et al., 1994) The results from this study can be used to improve current numerical models of granular gouge but can also directly be applied in improved seismotectonic scale models (Rosenau et al., 2017; Blank \& Morgan, 2019). The glass beads show a slip behaviour that naturally emerges from their frictional properties. This can be exploited for larger analogue models to model fault slip in a geometrically complex fault system. The range of available temporal and spatial scales, as well as the self-consistent scaling behaviour allow the application in many fields where rate-and-state friction is a dominant process such as landslides, glacial motion, mass movements and lithospheric deformation (Jerolmack \& Daniels, 2019). In comparison to numerical simulations the use of an analogue model allows to inherently link the spatial and temporal scales without having to rely on parametrization and grid based methods. The analogue approach allows to model small scale processes, such as earthquakes within a fault zone over many seismic cycles and over a much larger spatial scale within a shorter period of time than numerical simulations of similar complexity.

We here report characteristics of slip events in an analogue fault gouge consisting of spherical glass beads. In contrast to similar experiments of Frye and Marone (2002); Anthony and Marone (2005); Ferdowsi et al. (2013); Jiang et al. (2016); Cui et al. (2016) we explore the low pressure ( $\mathrm{kPa}$ instead of $\mathrm{MPa}$ ) regime which is rich in slip behaviours and generates regular stick-slip with more complete stress drops similar to seismic cycles along major faults in a highly reproducible and accessible way. Several studies established the large diversity in slip modes in such experiments. Changes in stiffness and normal stresses lead to first order changes in frictional stress, such as transition from stickslip to oscillation and stable sliding (Heslot et al., 1994). Nasuno et al. (1997) found localized precursor phenomena in thin sheared glass beads that precede large slip events. Moreover, the use of a ring-shear tester instead of commonly used direct shear apparatuses allows us to apply an in principle infinite amount of displacement and therefore a large number of events, which is a solid database for statistical analysis. Results from a similar apparatus by Cain et al. (2001) show that it is suitable to measure dilationcompaction cycles and show that the conditions in an annular shear cell lead to dilation during the loading phase and compaction during failure which is similar to the results obtained from rock mechanical tests in biaxial compression setups (Beeler \& Tullis, 1997).

For the same material we vary the extrinsic parameters normal stress $\sigma_{N}$, loading velocity $v_{L}$, and stiffness $k_{L}$. In this parameter space, we monitor the occurrence of slip events and creep, as well as the transitions from one slip mode to another. We characterize the analogue fault gouge with commonly used tests to derive the rate-and-state parameters, such as slide-hold-slide tests (SHS). We compare the findings to first order observations from rock friction experiments and assess the suitability of granular analogue fault gouge for its use in combined analogue and numerical modelling.

\section{Methodology}

To simulate fault behaviour in various settings we use a granular analogue modelling approach. Previous studies examined granular media under natural pressure conditions, whereas we are using conditions realized by analogue models, being 3 to 4 orders of magnitude lower (Rosenau et al., 2017). This prevents comminution of the glass beads and ensures constant frictional properties over the experimental duration, which gives well reproducible results. In a first step the data is analysed with simple methods to quantify basic properties which makes it possible to easily compare the results with previous works. The terminology for certain points and characteristics of the data is found in appendix Appendix A and Tab. A1. The data analysis is done using a suite of Python scripts that pick events and do statistic calculations. All of which are going to be avail- 
able as the open source software 'RST-Stick-Slipy' from the GFZ git repository (Rudolf, in prep) and are also included in the data publication (Rudolf et al., in prep).

\subsection{Rate-and-State Friction}

The relation between shear stress and normal stress for granular media and many other interfaces is determined by a non-linear combination of mean stress, slip velocity, stiffness and several non-dimensional parameters. This relationship is termed rate-andstate dependent friction that macroscopically leads to alternating cycles of slip, creep and locking, called stick-slip (Dieterich, 1978; J. H. Dieterich, 1979a; Ruina, 1983; Marone, 1998; Tullis \& Weeks, 1986; Beeler et al., 1994). This effect is used to describe and explain the various slip behaviours that are associated to earthquakes, e.g. slip on faults (Marone, 1998), earthquake nucleation (J. H. Dieterich, 1992) and slow slip events. In our study we use the relationships and testing procedures defined in Beeler et al. (2001), Marone and Saffer (2015) and J. Dieterich (2007) as well as adapted methodologies of Corbi et al. (2013), Bhattacharya et al. (2015) and Bhattacharya et al. (2017) to estimate the principal parameters for the rate and state equation. A short description of rateand-state friction and its application to our study is found in appendix A2.

To test which of the state evolution laws best describe our experimental data we take a semi-quantitative approach which considers certain observations, such as the evolution of stress during a hold phase, or the behaviour in unstressed SHS-tests. These results are then compared to other experimental findings. In some cases a close quantitative comparison is possible, while in others either the experimental setups are too different for a direct comparison, or an easily comparable quantity could not be found. E.g. the general dilatational behaviour in the reloading phase just after a hold period is similar for our experiments compared to the results by Beeler and Tullis (1997) which can then be qualitatively interpreted in the context of state evolution (Bhattacharya et al., 2017) (see section 4.2). Strong stick-slip effects, probably due to insufficiently high machine stiffness, prevented a direct fit of Eq. A1 to the data to estimate rate-and-state parameters from classical velocity stepping.

\subsection{Experimental Setup}

For the experiments we use a ring shear tester of type 'RST-01.pc' (Schulze, 1994; ASTM, 2016) which allows to shear a granular sample in an annular shear cell. The machine and methodology has been verified and calibrated using a standard bulk material (CRM-116 limestone powder) and is extensively used for characterizing granular materials in engineering and analogue modelling (e.g. Ritter et al., 2016a; Klinkmüller et al., 2016; Schulze, 1994).

The granular material is confined in a ring shaped shear cell and sheared against a lamellae-casted lid which also imposes the normal load (Fig. 1a+b). The normal load is adjusted using a motorized weight attached to a lever that pulls the lid from below. This ensures a constant normal load on the sample. Two bars attached to force transducers hold the lid in place and measure the shear forces acting on the lid.

The applied and resulting forces (normal and shear), driving velocity and vertical lid displacement are measured as individual channels at the analogue output of the machine. The main set of experiments were measured using a Peripheral Component Interconnect (PCI) based analogue-to-digital converter card (ADC) at a frequency of 12.5 $\mathrm{kHz}$ each (BMCM - PCI Base 50, controlled with BMCM Nextview ${ }^{\circledR}$ software). The measured values are averaged over 20 samples for noise reduction resulting in a final output frequency of $625 \mathrm{~Hz}$. Another set of experiments, mainly the slide-hold-slide (SHS) tests, were measured with a real-time embedded controller (NI - CompactRIO) at $50 \mathrm{kHz}$ per channel using an ADC module (C Series Universal Analogue Input Module, NI-9219, con- 
trolled by custom in-house software). This change was due to the end-of-life of the operating system during the course of this study which lead to hardware incompatibilities with the PCI-based approach. Similar to the other measurements, this data is averaged down to a frequency of $1 \mathrm{kHz}$. Based on the setup geometry, shear and normal forces are converted into shear and normal stresses according to ASTM (2016) and lid displacement into volumetric change (dilation/compaction). Shear forces are converted to shear stress using the moment of the crossbar $M_{d}=r_{s} \cdot F_{s}$, the median radius $r_{m}$ and the cross-sectional area of the lamellae $a_{d}$ (Eq. 1).

$$
\tau=\frac{M_{d}}{r_{m} \cdot a_{d}}
$$

The median radius $r_{m}$ is also the reference position at which the loading rate $v_{L}$ is defined because it divides the cell into two regions of equal volume. While the normal stresses are also continuously measured, we assume a constant normal stress that is equal to the value set at the start of the measurement. Due to internal correction factors which are not disclosed by the manufacturer there is always a slight discrepancy between set normal stress and measured normal stress. Partially, this discrepancy stems from the angle of the tie rods and the lid which exerts additional normal stress onto the sample (pers. comm. D. Schulze).

As granular material we use 300-400 $\mu \mathrm{m}$ sized fused soda-lime glass micro-beads supplied from Kuhmichel Abrasiv GmbH (Fig. 1c). They are characterized by a relatively low dynamic friction coefficient $(\mu=0.47)$ and no measurable cohesion $(C=$ $1 \pm 12 P a)$ as well as a strain hardening-weakening behaviour associated with dilationcompaction (Lohrmann et al., 2003; Klinkmüller et al., 2016; Ritter et al., 2016a). Glass beads are frequently used as a rock and gouge analogue material and generate stick-slip under laboratory conditions (e.g. Mair et al., 2002). Because they are non-cohesive we can approximate the instantaneous frictional resistance of the fault zone $\mu$ as the ratio of shear stress $\tau$ to the applied normal stress $\sigma_{N}$ :

$$
\mu=\frac{\tau}{\sigma_{N}}
$$

Before an experiment is started, the sieved samples are presheared by $10 \mathrm{~mm}$ at a loading velocity of $0.5 \frac{\mathrm{mm}}{\mathrm{s}}$ which ensures a fully developed shear zone without major post failure weakening (derived from Ritter et al., 2016a, 2016b). Tab. 1 lists the experimental parameters for the various tests performed for this study. For all tests (main and SHS) we use 4 different normal stresses of $5,10,15$, and $20 \mathrm{kPa}$. The major difference between the tests is the stiffness $k_{M}$ and loading rate $v_{L}$.

The main tests are conducted with logarithmically spaced velocity $v_{L}$ from 0.02 $\frac{\mathrm{mm}}{\mathrm{s}}$ to $0.0008 \frac{\mathrm{mm}}{\mathrm{s}}$. The duration of each run is the inverse of the respective loading velocity leading to equal displacement and a similar amount of slip events. Each individual test is carried out at constant normal load.

To limit the influence of stick-slip we perform the stressed SHS tests with maximum machine stiffness and at higher shear rates. To also estimate the rate effect on healing rate we vary the loading rate $v_{L}$ from 0.05 to $0.52 \frac{\mathrm{mm}}{\mathrm{s}}$. The hold times were increased logarithmically $t_{\text {hold }}=\{1 \ldots 1000\} s$ (in accordance with Eq. A7) and a constant load point displacement of $5 \mathrm{~mm}$ between the hold phases was applied. Additionally, we did one series of unstressed SHS tests with the same set of parameters as for the stressed SHS test but only with a single load point velocity of $v_{L}=0.16 \frac{\mathrm{mm}}{\mathrm{s}}$. For unstressed SHS tests we reduce the normal stress to zero during hold to evaluate whether the change in state $\theta$ is purely time dependent (Aging law) or shows a slip dependent behaviour (Slip law). Due to the time needed for the machine to unload and reload the normal stress, 


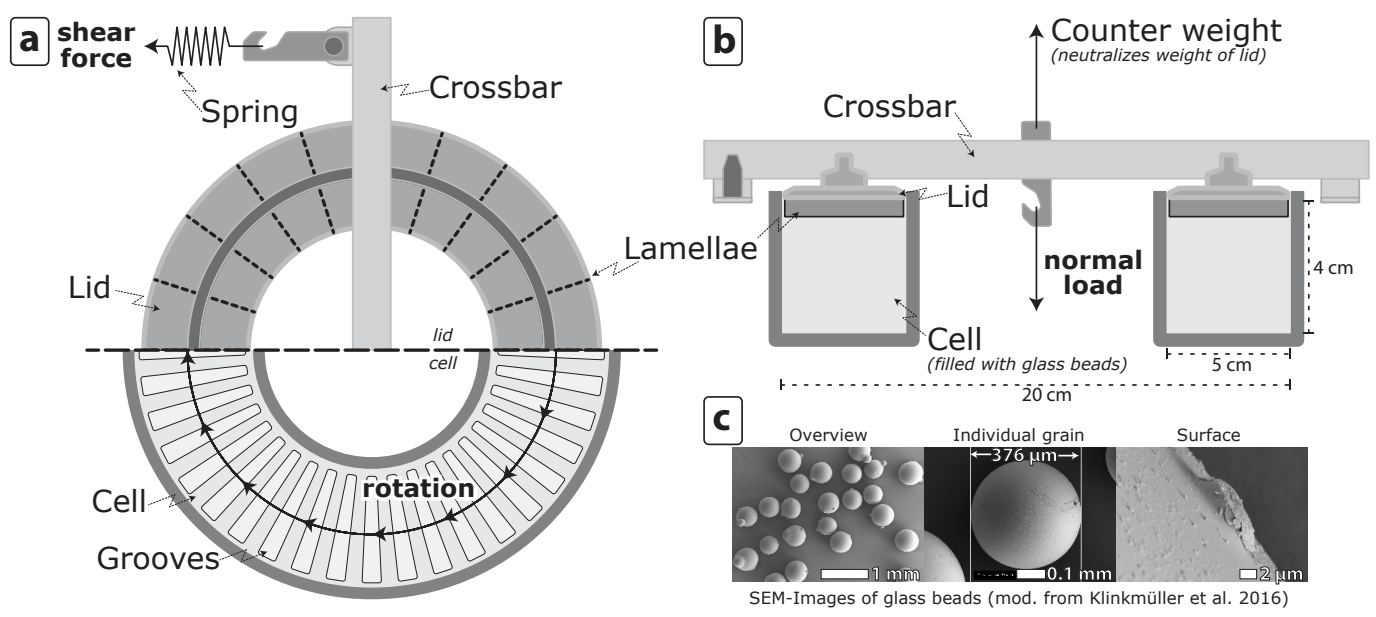

Figure 1. Schematic drawing of the modified ring shear tester. The system is loaded at loading velocities of $0.02 \frac{\mathrm{mm}}{\mathrm{s}}$ to $0.0008 \frac{\mathrm{mm}}{\mathrm{s}}$ by rotating the cell. The cell has grooves for a high friction interface which is mirrored by lamellae attached to the lid. A moveable weight pulls the lid from below by a motor driven lever for applying normal load. Force transducers behind the springs measure shear force. a) Top view the above part showing the lid and the bottom part showing the cell and its internal structure. b) Cross section through the whole setup. c) Scanning electron microscopy images of the glass beads showing the average particle size and the surface structures (modified from Klinkmüller et al., 2016).

which was a few seconds per $\mathrm{kPa}$, we only used hold intervals of $t_{\text {hold }}=\{100 \ldots 1000\} \mathrm{s}$. All SHS-cycles were repeated three times to be able to estimate the variance of the measurements.

\subsubsection{Adjustment to other setups}

To compare our experimental conditions with other setups in terms of stress conditions and stiffness we utilize the normalized stiffness $k_{N}$ and normalized loading rate $v_{N}$. Both have an influence on the material behaviour because of rate-and-state friction and are dependent on the setup. For our setup the normalized stiffness is calculated from the machine stiffness $k_{M}$, which is modified using springs, the geometrical factor $L_{M}$ converting shear force to shear strain and the applied normal stress $\sigma_{N}$.

$$
k_{N}=\frac{k_{M} \cdot L_{M}}{\sigma_{N}}
$$

From this the normalized loading rate $v_{N}$ is derived with the loading rate $v_{L}$ (Eq. 4). It can be interpreted as a non-dimensional stressing rate that describes the increase in stress counteracted by friction over time.

$$
v_{N}=k_{N} \cdot v_{L}
$$

For our setup the values for $k_{N}$ are in the range of $10^{-2}$ to $10^{2} \mathrm{~mm}^{-1}$ and for $v_{N}$ varies between $10^{-5}$ and $10^{-1} s^{-1}$. This is in the same regime as Nasuno et al. (1997) and at least three orders of magnitude higher than the values achieved by Beeler et al. (1994). 
Table 1. Experiment overview for this study

\begin{tabular}{c|c|c|c} 
Type & $\begin{array}{c}\text { Stiffness } \\
{[k]=\frac{N}{m m}}\end{array}$ & $\begin{array}{c}\text { Normal stresses } \\
{\left[\sigma_{N}\right]=P a}\end{array}$ & $\begin{array}{c}\text { Load point velocities } \\
{\left[v_{L}\right]=\frac{m m}{s}}\end{array}$ \\
\hline Main & $\{3.3$, & $\{5000$, & \\
tests & 19.6, & 10000, & $\{0.0008, \ldots, 0.02\}$ \\
$(\mathrm{RST})$ & 82.6, & 15000, & \\
& $1354.0\}$ & $20000\}$ & \\
\hline stressed & & $\{5000$, & \\
SHS & \multirow{2}{*}{1354.0} & 10000, & $\{0.05, \ldots, 0.52\}$ \\
tests & & 15000, & \\
& & $20000\}$ & \\
\hline unstressed & & $\{5000$, & \\
SHS & 1354.0 & 10000, & 0.16 \\
tests & & 15000, & \\
\end{tabular}

\section{Results}

We here describe the slip modes qualitatively (Fig. $2+3$ ) and quantitatively using the asymmetry of the event cycles (Fig. A2). Then we determine the constitutive parameters for our setup and analogue fault gouge which determine the stick-slip characteristics and slip behaviour by slide-hold-slide tests. To compare the data across the individual setups we use the normalized loading rate $v_{N}$ (Eq. 4) as a key parameter. This parameter contains the joint influence of normal stress and loading rate and makes it possible to plot all experiments together without major overlap. Nevertheless, in all cases there is a distinct influence of both parameters for the individual datasets so that in the following sections the results as a function of normal stress and stiffness are presented as well. We use a similar colour and marker code in most plots that show results from the experiments. Normal stress, in some cases loading velocity, is indicated by colour while the setup stiffness $k_{M}$ is indicated by markers. All errors in plots or in numeric values are given as twice the standard deviation $(2 \sigma)$ of the respective quantity. A rigorous error propagation is done during data analysis using the Python module 'uncertainties' (Lebigot, 2021).

\subsection{Slip Mode}

The slip mode is qualitatively defined by the evolution of stress during an experimental run (Fig. 2). Low stiffness leads to typical sawtooth shaped curves with very sharp acceleration immediately before failure (Fig. 2a $\mathrm{a}_{1}$ ). Increasing the stiffness increases the amount of pre-slip and slows the acceleration before failure. This is expressed as slightly smoother sawtooth curves but the duration of a slip event is still much shorter than the reloading phase (Fig. 2 $b_{1}$ ). For Spring $\mathrm{C}$ we find oscillations of weakly irregular shape (Fig. 2 $\mathrm{c}_{1}$ ). On average the increasing edge of an oscillation is a bit longer but only by a factor of 1.5 to 2 and not several orders of magnitude as for the softer springs. Another slip mode is observed for the highest stiffness which shows stick-slip cycles with a plateau of high stresses before failure. If the sample is at these high stresses we observe small and slower slip events that occur very close to failure (Fig. $2 \mathrm{~d}_{1}$ ). Figure 3 shows an overview of all qualitatively determined slip modes in all experiments. Furthermore, it indicates the full phase space in stiffnesses $k_{M}$ and loading velocities $v_{L}$ that was surveyed in this study. 

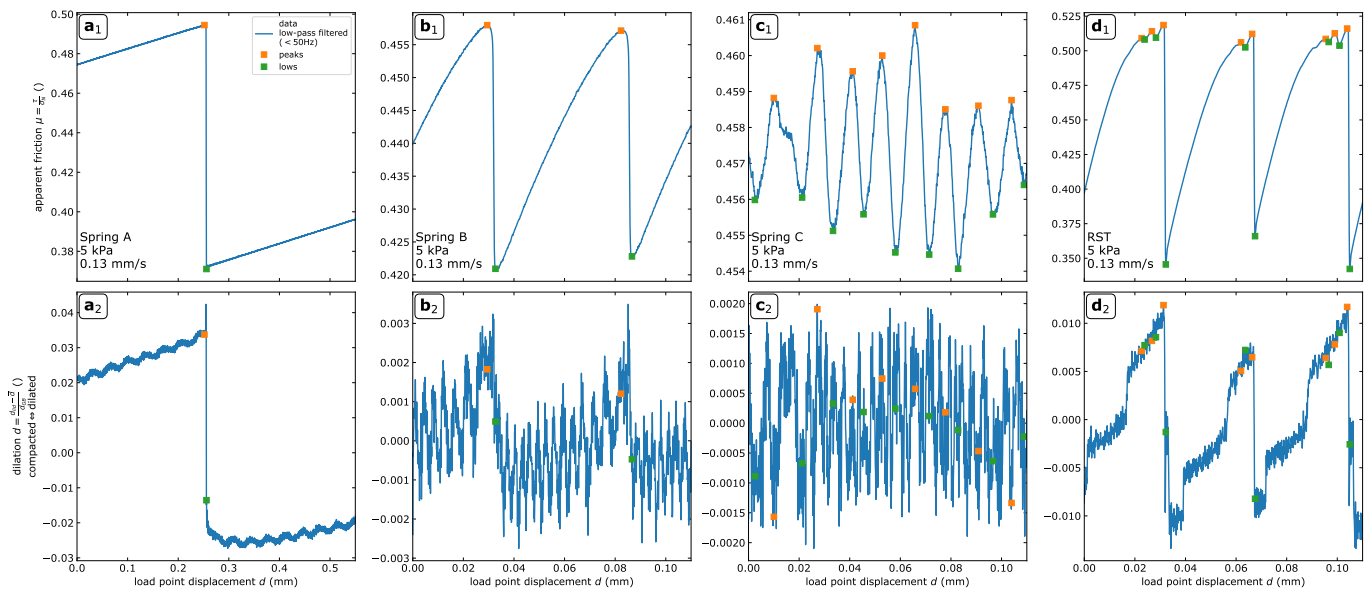

Figure 2. Exemplary stress and dilation curves during a typical experimental run. For all three experiments the normal stress and loading velocity are the same $\left(\sigma_{N}=5 \mathrm{kPa}, v_{L}=\right.$ $0.13 \frac{\mathrm{mm}}{\mathrm{s}}$ ) only the machine stiffness $k_{M}$ increases from top to bottom. a) Spring A - $k_{M}=$ $3.3 \frac{\mathrm{kN}}{\mathrm{mm}}$ : Regular sawtooth shaped stick-slip curve with a linear loading phase resulting from low stiffness. Due to the extremely high recurrence time this plot has been scaled down by a factor of 5 to be able to see a stick-slip event. b) Spring B $-k_{M}=19.6 \frac{\mathrm{kN}}{\mathrm{mm}}$ : Less sharp stick slip curves with a clear acceleration phase after peak strength. c) Spring C $-k_{M}=82.6 \frac{\mathrm{kN}}{\mathrm{mm}}$ : Oscillations due to intermediate stiffness. d) RST $-k_{M}=1354.0 \frac{\mathrm{kN}}{\mathrm{mm}}$ : Higher stiffness leads to non-linear loading behaviour and minor slip events just before major slip, while the loading velocity remains similar.

For all experiments we observe a characteristic succession of dilation and compaction. For perfect stick-slip, slip events lead to strong compaction of $\Delta d \approx 0.07$ grain diameters $d_{G B}$ which then slowly dilates during the interevent period (Fig. $2 \mathrm{a}_{2}$ ). In the first moments of failure for low stiffness experiments we often observe an initially dilating motion in the first few milliseconds. Experiments with stick-slip also show oscillations and characteristic patterns on a variable scale while experiments in the oscillating regime show a mostly random pattern of $\pm 0.001 d_{G B}$ (Fig. $2 \mathrm{c}_{2}$ ). For low stiffness experiments this oscillation is on a scale of $\pm 0.003 d_{G B}$ with a period of $2.5 \mathrm{~s}$. Increasing stiffness leads to a reduction of the oscillation period to values of $0.2 \mathrm{~s}$ for Spring B and <.1s for RST. With increasing amounts of creep we find additional oscillations in the dilation signal which gradually change their period closer to failure (Fig. $2 b_{2}$ ). For bimodal experiments, we observe a pattern of abrupt dilation events during the interevent period which is similar for consecutive interevent phases (Fig. $2 \mathrm{~d}_{2}$ ). The gradual change in oscillation period is also present for these experiments but on a smaller scale than for lower stiffness.

Quantitatively we describe the slip mode through the average asymmetry $r_{a}$ of the stick-slip curves and its distribution. Asymmetry $r_{a}$ is defined as the ratio of slip duration $t_{s}$ versus the reloading time $t_{r}$ (Fig. A2). We find that at generally low normalized loading rates $\left(v_{N}<10^{0} \mathrm{~s}^{-1}\right.$, low stiffness and low normal stress, Fig. 3a+b, Fig. A2a) the asymmetry is very high and has a low variability, although the dataset is relatively sparse in that region due to very long reloading phases $\left(t_{r e l}>10^{2} s\right.$, e.g. Fig. A2 $\left.\mathrm{a}_{3}\right)$. Pure stick-slip, dominant at low setup stiffnesses, has a very high asymmetry because the reloading phase is very long compared to the duration of an event (e.g. Fig. A2a). In general both experiments with low stiffness (Spring A and B), show relatively regular and well defined stick-slip events. This is indicated by relatively flat increases in shear stress and abrupt decays with strong to modest acceleration before failure (Fig. 2a+b). 


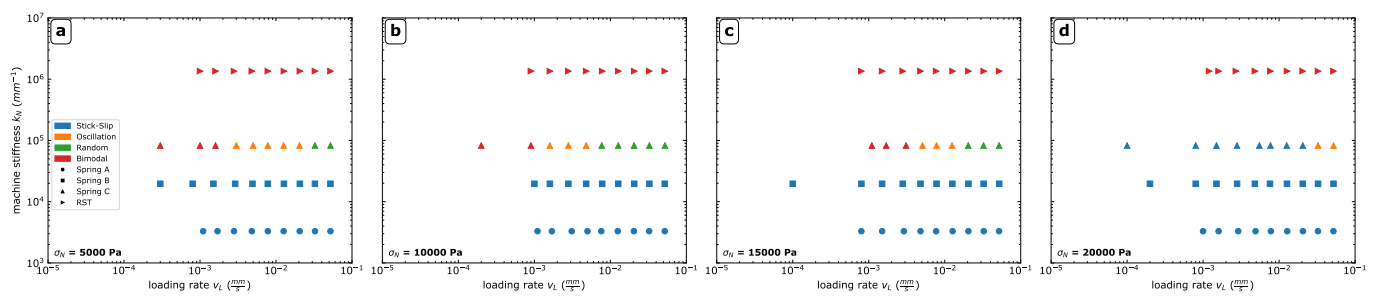

Figure 3. Qualitatively determined slip modes for all experiments in the full $k$ - $v$-space. All experiments for RST show a bimodal slip distribution and all experiments for Spring A and B show well defined stick-slip cycles. The intermediate stiffness for Spring C leads to variable slip modes depending on loading rate $v_{L}$ and normal stress $\sigma_{N}$. A transition from bimodal via oscillations to random is found for increasing loading rate. At higher normal stresses the bimodal slip mode is replaced by stick-slip and is also present at higher loading rates. Each column displays the result for a different normal stress. The legend applies to all subplots.

Experiments with Spring C show three different slip modes depending on normal stress and loading rate. With increasing loading rate we observe an evolution from bimodal over oscillation to random. This evolution is clearest for low normal stresses and less apparent than for high normal stress (Fig. 3). At normalized loading rates $v_{N}<$ $10^{0}$ the slip mode is bimodal with oscillating events preceding asymmetric events (Fig. 3a-c and e.g. Fig. A2 $c_{1}$ ). In the interval $v_{N}=\left\{10^{-0.5} \ldots 10^{0.5}\right\}$ we find low asymmetry with low variability which approaches $r_{a}=1$, which is the expression of oscillating events becoming more and more symmetrical. The asymmetry decreases until we find oscillating slip modes at normalized loading rates between 1 and $10 \mathrm{~s}^{-1}$. Oscillations are characterized by symmetrical increases and decreases of shear stress with an almost sinusoidal character (Fig. 2c). In terms of asymmetry this leads to an average ratio $r_{a} \approx$ 1 with a small variance (Fig. A2 $\mathrm{c}_{1-3}$ ). At higher normalized loading rate the system becomes random and the asymmetry shows a large variance with a mean asymmetry of $r_{a} \approx$ 1. Slip under these conditions tends to be chaotic and does not show any characteristic features.

At highest stiffness $k_{M}=1354.0 \frac{\mathrm{kN}}{\mathrm{mm}}$ (RST) we see a different evolution of slip mode with a complex sets that are influenced by normal stress and loading rate. For lower rates $v_{N}<10^{2}$ the events split into two different distributions one with high asymmetry and one with lower asymmetry (Fig. $\mathrm{A} 2 \mathrm{~d}_{2-4}$ ). As shown in Fig. 2d, slow and small events alternate with larger events in a characteristic sequence. The duality of slip modes leads to a bimodal distribution of asymmetry with a distinct separation of small/slow and large/fast events. Normal stress has a strong influence on how well defined the separation between these two is. High normal stress leads to a clear separation which is at least one order of magnitude. At higher rates continuous distribution is observed while retaining a mean that is larger than 1 , which still indicates defined stick-slip cycles rather than randomness.

In general, we find that above a certain stiffness the slip mode switches from simple stick slip (Spring A+B) to a more complex pattern of slip modes. Furthermore, high loading rates suppress the evolution of well defined stick slip cycles and lead to oscillations and random slip modes. Normal stress defines the behaviour for low loading rates, which is most apparent at higher stiffness (Spring C + RST). At low normal stress, the slip mode is mainly bimodal which is due to higher amounts of creep. Increased normal stress suppresses creeping mechanisms and forces a change from bimodal slip mode, with slow events to pure stick slip. Additionally, this leads to a shift in mode space so that oscillations occur at higher loading rates (Fig. 3a-c vs. d). 


\subsubsection{Stiffness}

The setup has two types of stiffness, one with and one without sheared material. The latter is straightforward to measure by fixing the lid to the shear cell and measuring the force increase while moving the shear cell. The basic stiffness of the apparatus $k_{M}=1354.0 \frac{\mathrm{kN}}{\mathrm{mm}}$ (RST) is mainly influenced by the stiffness of the load cells that measure shear force which acts in series with the aluminium of the lid, tie rods and crossbar. Adding springs in between the load cells and tie rods lowers this stiffness to the values reported in Tab. 1 (Spring A $-k_{M}=3.3 \frac{\mathrm{kN}}{\mathrm{mm}}$, Spring B $-k_{M}=19.6 \frac{\mathrm{kN}}{\mathrm{mm}}$ and Spring $\left.\mathrm{C}-k_{M}=82.6 \frac{\mathrm{kN}}{\mathrm{mm}}\right)$.

For the types of tests reported in this study another type of stiffness is of relevance, the reloading stiffness $k_{L}$ which is a combination of machine stiffness $k_{M}$ and material stiffness. This property is calculated from the linear part of the reloading phase between slip events. We find that for experiments with low machine stiffness (Spring A+B) the normalized reloading stiffness $k_{R}$ is roughly one order of magnitude smaller than the normalized machine stiffness (Fig. 4). For Spring B there are a few outliers and a weak increase in $k_{R}$ is observed due to the influence of normal stress for the lowest normal stress $\sigma_{N}=5 k P a$. Spring $\mathrm{C}$ shows different results depending on the type of events considered. If only dynamic events with a slip rate above a critical threshold (comp. section 3.1) are considered (Fig. 4a) the reloading stiffness $k_{L}$ is one order of magnitude smaller than the machine stiffness only for high normal stresses $\left(\sigma_{N}=20 k P a\right)$, for lower normal stresses $\left(\sigma_{N}<15 k P a\right)$ the difference is reduced to only half an order of magnitude. Considering all events (Fig. 4b) we see that especially for these lower normal stresses there is an influence of loading rate, that is apparent from the large spread in values due to variable slip modes (Sec. 3.1). The strongest difference is measurable for the highest machine stiffness (RST) where $k_{R}$ is roughly 1.5 orders of magnitude smaller, with only a minor increase in variability for all events.

\subsection{Event Magnitudes}

Comparing the frictional stress drops $\Delta \mu$ for all experiments we find three different groups of stress drop highlighted in Fig. 4c. These are distinguished by their magnitude and variability of stress drop, as well as the evolution with increasing normalized loading rate.

The first group occurs at low to medium normalized loading rate and at high stress drops (red area in Fig. 4c). The stress drop shows an exponential decrease with a similar slope for most experiments in this region. It consists mainly of fast slip events and experiments at low to intermediate stiffness (Spring A, B and C). A minor outlier is the Spring A-5 kPa experiment which has slightly higher stress drops but the same slope.

The second group consists exclusively of fast events at the highest stiffness (blue area in Fig. 4c). They all plot at high normalized loading rate and show the highest stress drops which is roughly one order of magnitude higher than for the previous group at similar normalized loading rate. The evolution of stress drop shows a different slope, but is also decreasing with increasing normalized loading rate.

The third group are slip events with a low stress drop $\Delta \mu<10^{-2}$ and a large variability in stress drop that may span one or two orders of magnitude (green area in Fig. 4c). This group is dominated by slow events. In general, the stress drop is decreasing for increasing normalized loading rate, but the slope is not constant.

\subsection{Slip Velocities}

From the above observations we find that there is a characteristic difference between certain events under certain conditions leading to a bimodal distribution of asymmetry. 

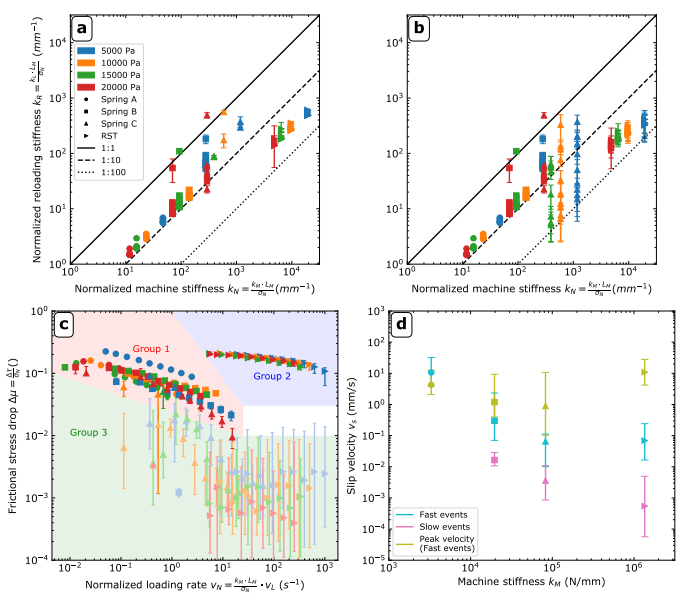

Figure 4. a+b) Measured reloading stiffness $k_{L}$ in comparison with machine stiffness $k_{M}$ for reloading phases of a) fast events and b) all events. The reloading stiffness $k_{L}$ is one order of magnitude smaller than the machine stiffness (dashed line). c) Frictional stress drop $\Delta \mu$ distributions for all experiments. Each point represents the median and the error bars enclose $95 \%$ of all values. Solid colours highlight events which are found to be 'fast' events, 'slow events' are shown in lighter colour. The coloured areas define the individual groups that were identified. d) Average slip velocity during an event for fast events and slow events. The experiments with lowest stiffness (Spring A) show only fast events and the slow event data point for Spring B is based on a single experiment.

This difference is highlighted using the average slip velocity $\overline{v_{s}}$ during an event as an indicator (Fig. 4d). The fastest slip events with $v_{s} \approx 10 \frac{\mathrm{mm}}{\mathrm{s}}$ are observed for the lowest stiffness (Spring A, $k_{M}=3.3 \frac{\mathrm{kN}}{\mathrm{mm}}$ ) which is mainly due to the much larger slip during an event. With increasing stiffness the fast slip velocity is decreasing to a level of $10^{-0.5}$ to $10^{-1} \frac{\mathrm{mm}}{\mathrm{s}}$. When slow events are present, which is not the case for all experiments at intermediate stiffness (Spring $\mathrm{B}+\mathrm{C}$ ), they are generally one to two orders of magnitude slower than the fast slip events $\left(v_{s} \approx\left\{10^{-2} \ldots 10^{-3}\right\} \frac{\mathrm{mm}}{\mathrm{s}}\right)$. The difference between fast and slow events increases towards the highest stiffness experiments, which also has the highest variability for slow slip velocities. Additionally, the peak slip velocity, that is the highest instantaneous slip velocity during a slip event, increases with increasing stiffness. The peak slip velocities are generally higher or in the same range as the mean slip velocity for fast events. Towards higher stiffness, the peak slip velocity is 2 orders of magnitude higher than the average slip velocity during a fast event. Typical peak slip velocities are in the range of $v_{S}=1 \ldots 10 \frac{\mathrm{mm}}{\mathrm{s}}$.

These slow events are characterized by low stress drop, low stress drop rate and a characteristic occurrence late in the cycle at generally high mean stress (Fig. 5). The relative amount of slow events decreases with increasing normal stress. For low normal stress more than $40 \%$ of the total events are found to be slow events, whereas for higher normal stresses it is $5-10 \%$. Additionally, there is a variation in occurrence with loading velocity. At high loading velocity only very few slow events are detected, while at low loading velocity multiple slow events of increasing size can occur before one main event.

We find that in the series with Spring $\mathrm{C}\left(k_{M}=82.6 \frac{\mathrm{kN}}{\mathrm{mm}}\right)$ at low normal stress and low loading rate these events show a nearly oscillating pattern of multiple cycles that is occasionally perturbed by a fast slip event. This is also highlighted in the asymmetry 

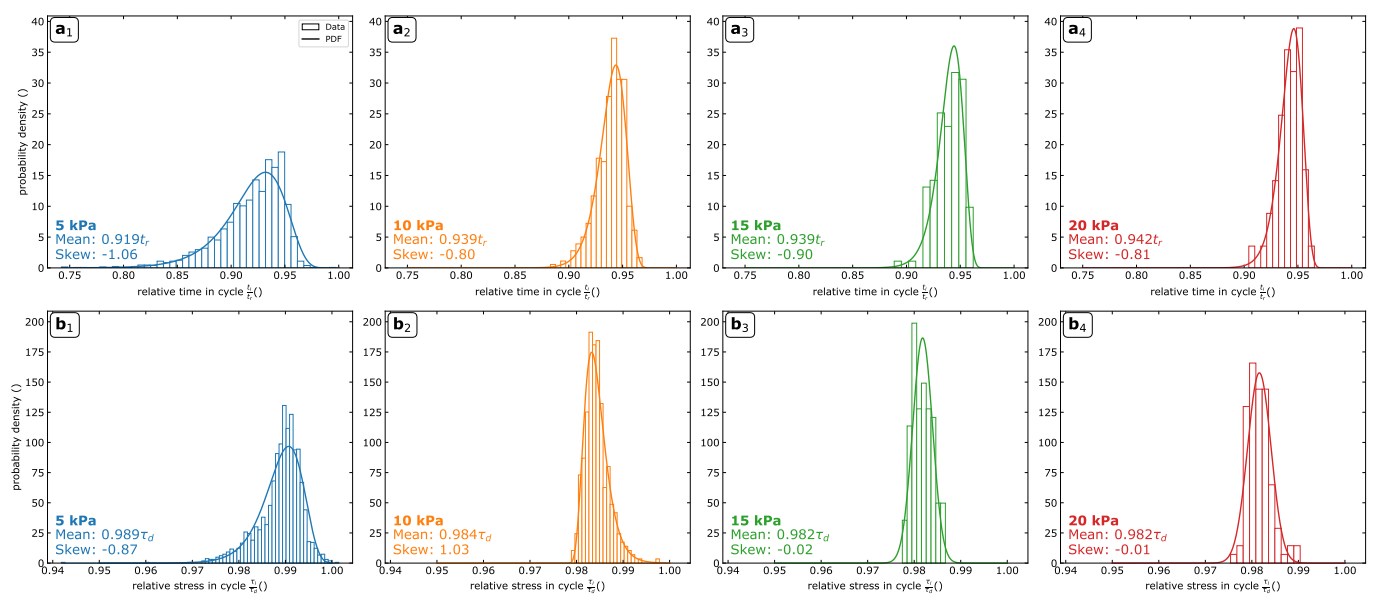

Figure 5. Timing and stress level of slow events during experiments of the highest stiffness $\left(\mathrm{RST}, k_{M}=1354 \frac{\mathrm{kN}}{\mathrm{mm}}\right)$. a) Relative temporal occurrence of slow slip events. The probability increases towards failure with a maximum of $0.95 t_{r}$ and very few events before $0.90 t_{r}$ and after $0.96 t_{r}$. b) Stress level where the slip events occur. The events are almost normal distributed with maxima between $0.98 t_{r}$ and $0.99 t_{r}$.

where these experiments have a bimodal distribution with one mode at high asymmetry (fast events) and one mode at low asymmetry (slow events). For the highest stiffness (RST, $k_{M}=1354.0 \frac{\mathrm{kN}}{\mathrm{mm}}$ ) the slip rates are similar to Spring B for the fast events, but the slow events are slightly slower and show a higher asymmetry. There are fewer slow events and of smaller magnitude, with an average stress drop that is only $2.6 \%$ of the corresponding main event.

The occurrence of slow events shows a specific temporal pattern for the highest stiffness. The temporal distribution of slip events show a log-normal distribution skewed towards the end of the cycle and they do not occur in the first half of a cycle. The probability of occurrence increases from $0.7 t_{r}$ onwards with a mean of $0.92 t_{r}$ to $0.94 t_{r}$ and peaks at $\approx 0.95 t_{r}$ (Fig. 5a). Then the probability drops abruptly to zero and for all experiments almost no precursor has been detected in the last moments of a cycle. Higher normal stresses shift the onset of occurrence closer to failure with a smaller variability but still with no events immediately before failure. The stress level at which the slow events occur is generally very close to the stress level of the main event (Fig. 5). The curves show a log normal distribution for low normal stresses which changes to a normal distribution (skew $\approx 0$ ) at higher stress level. For higher normal stresses the slow events occur around $0.98 \tau_{d}$, and for $\sigma_{N}=5 \mathrm{kPa}$ at higher levels of $0.99 \tau_{d}$. Again we see a strong increase in occurrence up to a certain level of stress and an absence of events at stress levels very close to failure strength $\left(>0.99 \tau_{d}\right)$.

\subsection{Interevent times}

In experiments where a unimodal distribution of asymmetry is found, it is straightforward to define the interevent time as the time between the individual events. But for bimodal distributions it is more complex. Therefore we use the term 'recurrence time $t_{r e c}$ ' for the time between any events (denoted by ${ }_{i}$ ) and the term 'reloading time $t_{r e l}$ ' for the time between the fast events (denoted by ${ }_{d}$ ). This results in the following definitions: 


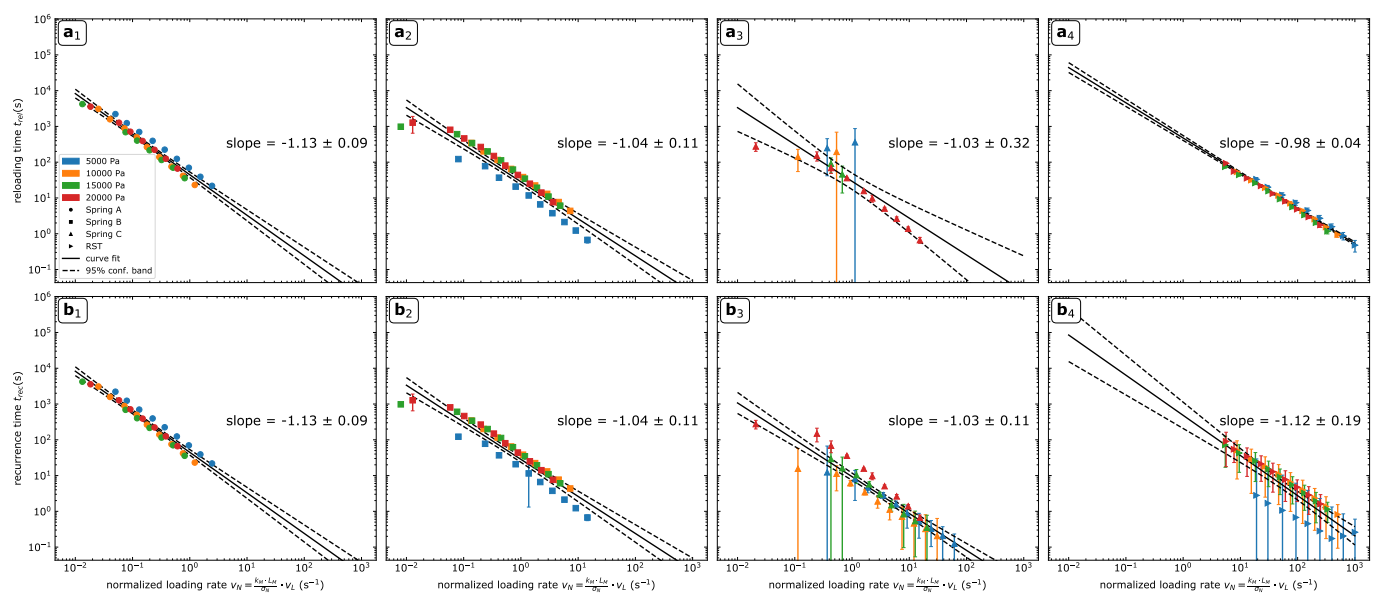

Figure 6. a) Reloading and b) recurrence times in comparison with normalized loading rate. The exponent is significantly different from $n=-1$ for the lowest stiffness $\left(\mathrm{a}_{1}\right.$ and $\left.\mathrm{a}_{2}\right)$ which means that the recurrence decreases stronger than expected by the increase in normalized loading rate. The other stiffnesses show exponents that are only slightly smaller than $n=-1$ with larger errors.

$$
\begin{aligned}
& t_{r e c}=t_{i}-t_{i-1} \\
& t_{r e l}=t_{d}-t_{d-1}
\end{aligned}
$$

In general the interevent times decrease with increasing normalized loading rate in an exponential fashion. The interevent times $t_{r e c}$ and $t_{r e l}$ are essentially the same for low stiffness setups (Spring $\mathrm{A}+\mathrm{B}$ ) because there is only one experiment in these series with slow events. For Spring $\mathrm{C}$ there are only few fast events which leads to a large error for the reloading times (Fig. 6a $\mathrm{a}_{3}$ ). Furthermore, there is a strong influence of slow event on the variance of recurrence times for the highest stiffness (RST, Fig. 6 $\mathrm{b}_{4}$ ). The power law exponent is slightly lower than $n=-1$ which means that there is a stronger decrease in reloading or recurrence time than what would be expected if there would be a direct correlation. Only the evolution of reloading time, that is for fast events, for the highest stiffness shows a power law exponent of $n=-0.98 \pm 0.04$ which indicates that the occurrence of fast events is directly proportional to the normalized loading rate.

\subsection{Rate-and-State Parameters}

The healing rate $b$ is determined from the change in peak stress after increasingly larger hold times (Eq. A6). We find that all stressed SHS-tests show a positive healing rate (Fig. 7a). The mean healing rate from all fits is $b=0.0057 \pm 0.0005$ which indicates time-dependent strengthening of the granular fault over time. There is no apparent correlation of healing rate $b$ with loading velocity $v_{L}$ (Fig. $7 \mathrm{c}$ ). However, we observe a higher healing rate for a low normal stress of $\sigma_{N}=5 k P a$. Statistically it is not significantly different in the $95 \%$ confidence band in comparison with the other series due to a relatively high error for all fits at this normal stress $\left(\overline{b_{5 k P a}}=0.007 \pm 0.003\right.$, Fig. $\left.7 \mathrm{a}_{1}\right)$. But the individual $b$ values all plot outside the $95 \%$ interval of the mean fit of all data sets combined (black dotted line in Fig. 7d). 

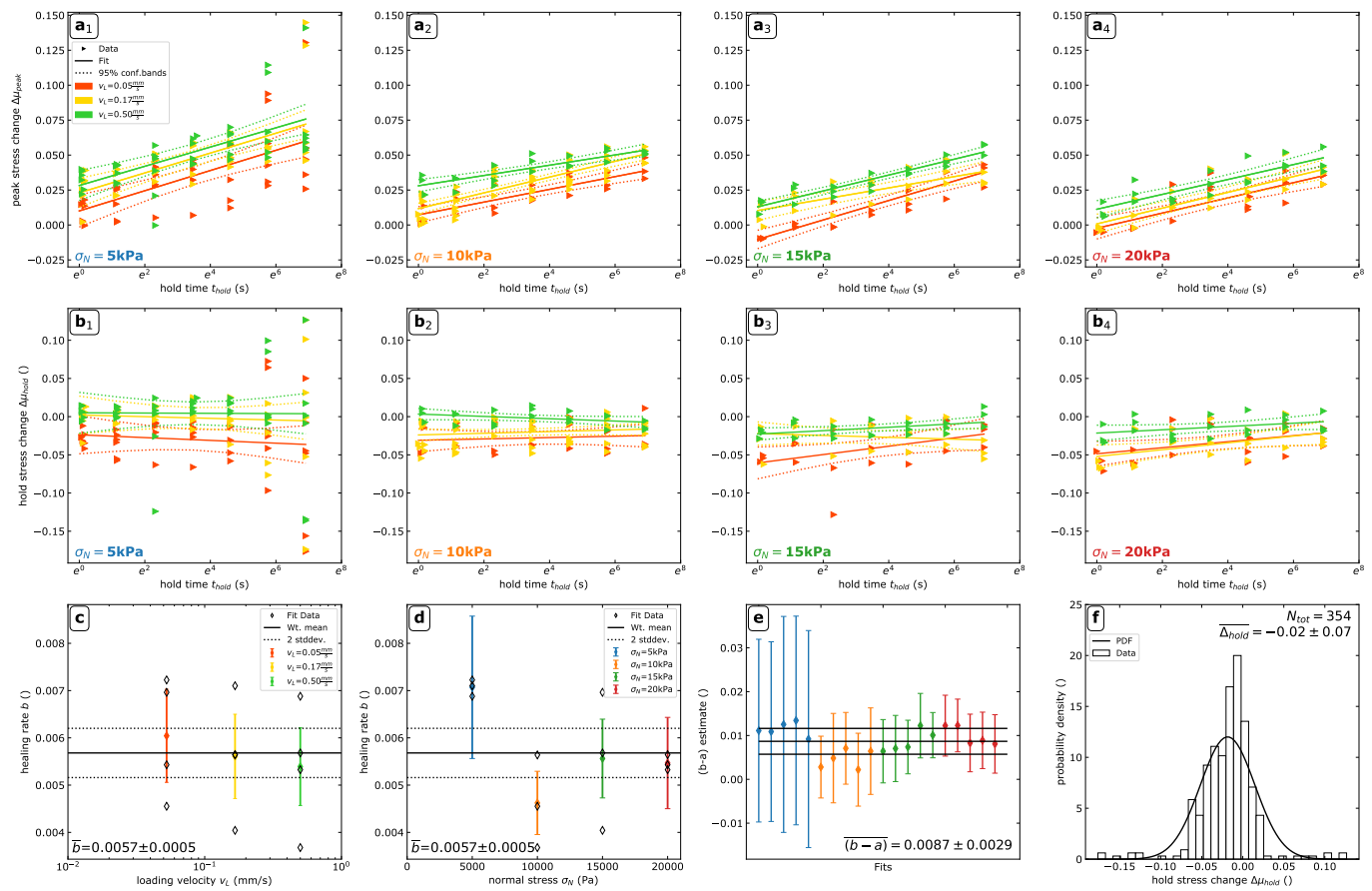

Figure 7. Overview of all slide-hold-slide related tests and quantities. a a $1-4$ ) Change in peak stress $\Delta \mu_{\text {peak }}$ after a hold interval $t_{\text {hold }}$ compared to the average pre-hold level during sliding. The slope of the log-linear fit is the healing rate $b$ which is positive for all experiments. The legend in $a_{1}$ applies to plots $a_{1-4}$ and $b_{1-4}$, the errors given are derived from the covariance of fit $\left.\left(2 \sigma=2 \sqrt{s^{2}}\right) . \mathrm{b}_{1-4}\right)$ Change in hold stress $\Delta \mu_{\text {hold }}$ during a hold interval due to creep. c) Synthesis of all fits for healing rate $b$ from $a_{1-4}$ with respect to loading velocity. The fitting errors on the 'Fit Data' points have be hidden for better visualization but are included in the error of the mean through error propagation with weighted averages. d) Synthesis of all fits for healing rate $b$ from $\mathrm{a}_{1-4}$ with respect to normal stress showing anomalously high values for $\sigma_{N}=5 k P a$. The errors in this plot are displayed in the same way as for c). e) Estimation of ( $b \quad-\quad a)$ from subsets of the experiments in $a_{1-4}$ sampled according to Eq. A7. The legend is the same as in d). f) Histogram of all hold stress changes $\Delta \mu_{\text {hold }}$ from $\mathrm{b}_{1-4}$ showing a normally distributed change which is not significantly different from $\Delta \mu_{\text {hold }}=0$ due to the high error. 
The direct effect $a$ is derived from two approaches. The first uses the offset in yaxis intersect of the peak stress change $\Delta \mu_{\text {peak }}$ (Eq. A8). This effect is clearly visible in Fig. 7a where the average peak stress change increases consistently for increasing loading rates while the slope stays constant. From the average increase in peak stress change with increasing loading velocity we compute a direct effect $a=-0.0074 \pm 0.0031$. As a result we calculate a first $(b-a)=0.0131 \pm 0.0031$ from this observation only. The second approach exploits the selection of loading velocities with respect to the hold times so that Eq. A7 is fulfilled (after Beeler et al., 2001). The average $(b-a)=0.0087 \pm$ 0.0029 fitting all possible combinations from all experiments (Fig. 7e). Using $b$ from the previous estimate we arrive at a direct effect $a=-0.0030 \pm 0.0030$ which is less than the previous estimate.

Another important observation for rate-and-state friction is the change of stress during the hold phase $\Delta \mu_{h}$ old (Fig. $7 \mathrm{~b}_{1-4}+\mathrm{f}$ ). The dataset is very noisy for this observation. We observe a weak correlation of hold stress change over time with increasing normal stress. At low normal stress $\left(\sigma_{N}=5 k P a\right.$, Fig. $\left.7 b_{1}\right)$ the data set shows a negative slope which becomes smaller at $\sigma_{N}=10 \mathrm{kPa}$ (Fig. $7 \mathrm{~b}_{2}$ ) and changes to a positive slope for $\sigma_{N} \geq 15 k P a\left(\right.$ Fig. $\left.7 \mathrm{~b}_{3+4}\right)$. However, the estimated errors for these fits are quite large and while on average the hold stress change is negative $\Delta \mu_{h}$ old $=-0.02 \pm$ 0.07 it is not significantly different from zero (Fig. $7 \mathrm{f}$ ).

\subsubsection{Additional Observations from Main Experiments}

We observe an increase in peak strength with increasing reloading time for the main experiment series. Plotting the reloading time $t_{r e l}$ against peak frictional strength at failure $\tau_{p}$ a log-linear increase can be observed (Fig. 8a). The observed slope ranges from $\beta=0.0083$ to $\beta=0.0130$ and indicates a time- or rate-dependent healing with a similar order of magnitude as the healing rate $b$. In addition, we observe a decrease in average frictional strength $\bar{\mu}$ with increasing loading rate $v_{L}$ (Fig. 8b). The average slope of all four stiffnesses is negative ranging from $(\alpha-\beta)=-0.0027$ to $(\alpha-\beta)=-0.0067$ indicating velocity weakening conditions. The extremely low error for the individual fits is the result of the large amount of data points for each experiment ( $i 10$ million) because the complete time series is used for fitting. This drastically narrows the confidence band for the slope.

Furthermore, comparing the reloading time $t_{r e l}$ with the loading velocity $v_{L}$ we find a power-law dependency with exponents $B>-1$ (Fig. 8c). This shows a longer reloading time than extrapolated for the simple increase in loading velocity which is another indicator for time- or rate-dependent healing.

\section{Discussion}

\subsection{Similarity of Constitutive Parameters}

In rate and state friction three key parameters are determined, the direct effect $a$, the healing effect $b$, and the state evolution variable $\phi$ (J. H. Dieterich, 1979a; Marone, 1998). From our type of experiments we can not observe the evolution of friction directly because our system is inherently unstable. This is due to the system stiffness $k_{M}$ which is below the critical stiffness $k_{c}$.

The healing rate $b=0.0057 \pm 0.0005$ which is equivalent to a frictional strengthening rate $\beta=0.0122 \pm 0.0005$ in $\log _{10}$-space is at the upper estimate of natural faults and fault rocks (e.g. Alpine Fault or Scheggia Fault in Carpenter et al. (2016) or other data in Marone et al. (1990); Marone (1998)). This means that the analogue fault material shows a similar amount of time-dependent healing that is observed for natural samples in rock mechanical tests. The underlying physical process is different for analogue 

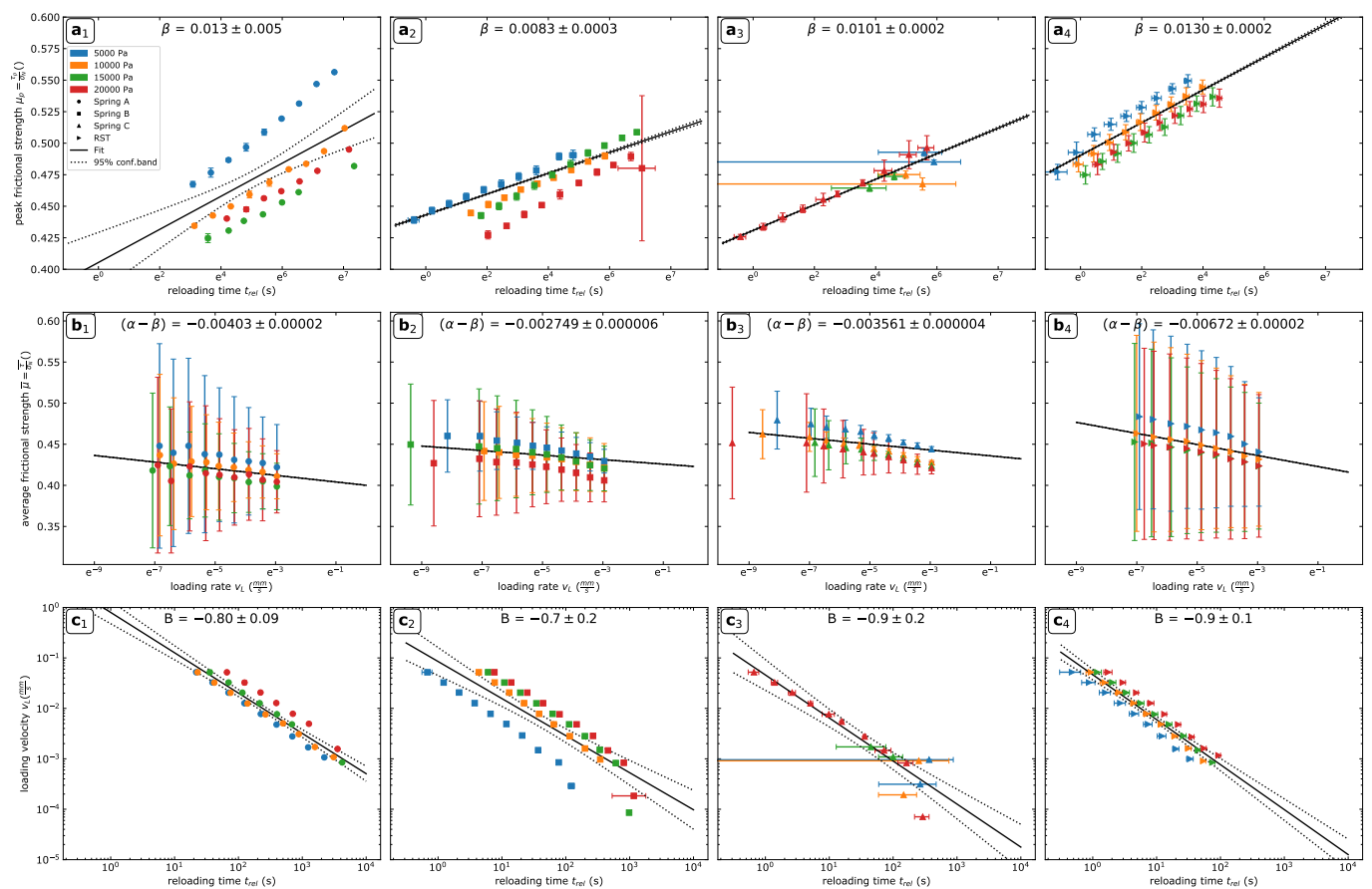

Figure 8. a) Change in peak stress with longer reloading time, which is the time between large slip events. The slope $\beta$ of the log-linear fit is similar to the healing rate $b$ from SHS-tests. b) Change in average frictional strength depending on loading rate. The slope $\alpha$ is an approximation of the rate-and-state parameter $(a-b)$. c) Loading velocity $v_{L}$ compared to reloading time $t_{r e l}$. A negative power-law coefficient that is larger than -1 highlights longer reloading times than normal. The legend in $\mathrm{a}_{1}$ ) applies to all subplots, the confidence band is derived from the covariance of fit $\left(2 \sigma=2 \sqrt{s^{2}}\right)$. 
materials, although a certain amount of granular mechanical strengthening due to grain rearrangement is probably also present in a dry natural fault. Therefore, the glass beads are found to be usable for small-scale seismotectonic models under analogue modelling conditions. The change of frictional strength over time and average frictional strength are similar to a natural fault and can be used to simulate seismic cycles with dynamic similarity. Due to the higher healing, which is $\approx 30 \%$ higher than most rocks, the analogue seismic cycles can be shorter in comparison with natural examples in order to represent a scaled model.

We observe a negative direct effect $a=-0.0074 \pm 0.0031$ which is not realistic but is needed to match the value of $(b-a)=0.0087 \pm 0.0029$ at such high healing rates. Furthermore, the friction during hold is higher $\left(\frac{\mu_{h}}{\mu_{0}}>1\right)$ just after a hold phase starts that indicates $a<0$ because for $a>0$ and $\frac{v_{L}}{v_{0}}<1$ we would expect that $\frac{\mu}{\mu_{0}}<1$. Because of the presence of creep in the granular shear zone we assume that initially the loading rate is not zero but very small so that $\frac{v_{L}}{v_{0}} \ll 1$ (see also Sec. 4.2 ) so we could see an effect similar to a large negative velocity step. This is also due to finite machine stiffness $k_{M}$ which was also observed by Marone and Saffer $(2015)$. The value of $(b-a)<$ 0 indicates velocity weakening which results in instability under our conditions that is confirmed by the stick-slip cycles in the other experiments. Direct fitting of velocity stepping data to get clearer results was not possible because the machine stiffness was not high enough to produce real steady state slip at our conditions.

Assuming that the change in peak frictional strength $\mu_{p}$ with increasing reloading time $t_{r e l}$ (Fig. 8) is similar to the healing rate $b$ we find that in the main experiments the healing rate $b \approx 0.0111 \pm 0.0011$ and $(a-b) \approx-0.004266 \pm 0.000007$ which yields a direct effect $a \approx 0.0068 \pm 0.0011$. These values are in the same order of magnitude as the other estimates but show a positive direct effect. These observations however include creep and transient slip events during the reloading phase which influences the estimate of $b$. The estimated weakening $(a-b)$ additionally includes the effect of the stick-slip cycles which distort the calculation of the mean friction. Overall, the values are in the same order of magnitude as the estimates from our SHS-tests and agree well with the above literature values.

In addition, by qualitatively matching rate-and-state parameters to our SHS-tests we find that the critical slip distance $D_{c}$ is in the order of $10^{-1} \mathrm{~mm}$. Direct fits of the SHS-Tests obtained a $D_{c} \approx 200 \mu m$, by assuming an extremely low loading velocity $\left(v_{L}=\right.$ $10^{-324} \frac{\mathrm{mm}}{\mathrm{s}}$, smallest float represented in NumPy) during hold to obtain valid results during the hold phase. But because the results for the other parameters $a$ and $b$ by direct fitting using non-linear least squares were not stable, these approximations are not statistically sound. Nevertheless, these values are reasonably close to values found in rock mechanical tests which vary from 2 to $100 \mu m$ (J. Dieterich, 2007, and references therein).

There is no statistically significant difference in the estimate of $(b-a)$ from soft and stiff systems, as expected for a material property. The scaling of strength at the onset of slip is consistent with the findings of Beeler et al. (2001) who show the same type of scaling. The scaling coefficient typically attributed to natural rocks or gouge in the seismogenic zone, is in the same range (0.011 to 0.015 (Beeler et al., 2001); $\approx 0.01$ (Scholz, 1998); 0.001 to 0.01 (J. Dieterich, 2007)). Other analog model studies have used (ba) values in the same range to model seismotectonic processes with other materials (gel on sand paper: 0.028 (Corbi et al., 2013); rice: 0.015 (Rosenau \& Oncken, 2009); cacao, ground coffee, and others: (Rosenau et al., 2017)). Therefore, we consider our models to be dynamically similar to the natural prototype, to rock deformation experiments in the MPa-range (e.g. Tullis \& Weeks, 1986), and to numerical simulations of rate and state friction (e.g. Ferdowsi et al., 2013). 


\subsection{State Evolution During Hold Phases}

During a hold phase the state $\theta$ of the system changes according to a certain relationship (section A2) which leads to a change in frictional strength of the fault. Beeler et al. (1994) state that purely time-dependent healing, which is given by the Aging law (Eq. A2), is independent of stiffness while the Slip law (Eq. A3) shows a dependency on stiffness because it requires active fault slip during healing. For our SHS-experiments we did not systematically vary stiffness but a change with normal stress was observed. The experiments at lowest normal stress show a higher than average healing rate. Additionally, these experiments show a decrease in stress during the hold phase. We attribute this to enhanced creep which is promoted by the low normal stress. As a result, the healing is amplified by larger amounts of slip in our experiments. In terms of constitutive laws this would mean that our material is better characterized by the Slip law than by the Aging law. A simple experimental test is to use additional data from unstressed SHStests (Marone, 1998). However, preliminary experiments with unloading showed that it is technically not feasible to do unstressed tests with our testing apparatus because the loading and unloading of the samples take too much time in comparison to the hold durations. We observed less healing for these tests but the dataset is very noisy so the results are statistically not relevant.

For most experiments we observe an upwards step in stress immediately after the onset of holding which indicates a negative $a$ which is also evident from the estimates of $(b-a)$. The step is followed by an exponential decay to a lower residual stress which shows a decay rate $\lambda=2.1 \pm 1.2 s^{-1}$. The stress decays to the residual stress after hold within less than 3 seconds ( $<1 \%$ difference). This indicates that creep due to shear stress quickly dissipates and the sample is not slipping along the shear surface during hold. The thickness of the granular packages first oscillates at a frequency of around $19 \mathrm{~Hz}$ and stabilizes to a constant value within the same time as the shear stress. These observations indicate that there is no measurable slip during hold which means that slip during hold is minute and therefore the Aging law is more appropriate. In spite of many points that speak for the Aging law, according to Bhattacharya et al. (2017) it is not sufficient to only look at the evolution of stress during hold phases but also to model the experimental data numerically. Therefore, we cannot exclude the possibility that other state evolution laws apply and additional experiments with different stiffnesses and numerical modelling of the actual data are needed to clarify this finding.

\subsection{Micromechanical processes}

Granular material gains shear strength due to force chains oriented in the direction of the maximum stress (Cates et al., 1998). Depending on the number, length and orientation distribution of such chains shear deformation might be stable or unstable. Stick-slip is therefore interpreted as a cyclic setup and breakdown of force chains, the frequency and size of which should be a function of grain size distribution (Mair et al., 2002). Furthermore, granular materials exhibit so called 'jammed states', where jamming is induced at high packaging density or by application of shear stress (Bi et al., 2011). We corroborate this view as large slip events are associated with compaction while the interseismic period is characterized by accelerating creep and dilation (Figure 2).

The normal stress is one of the critical factors that control the creep threshold of the system. For low normal stresses it is easier for the grains to rearrange during the creep phase. Firstly, this results in higher background slip of grains that exhibit a much lower normal stress along their contacts and can easily slide along each other. Secondly, the ratio of normal stress to dilatational stress, that pushes the grains apart when sliding over the rough internal shear zone, is smaller. Therefore, the force chains are less effective in strengthening the material at low confining pressures. 
The occurrence of small slip events is in accordance with other studies that show transient effects during the transition of the stick phase to dynamic slip (Nasuno et al., 1998; Ferdowsi et al., 2013). Because they are much smaller than the main events it is suggested that the events are the expression of internal reorganization in the granular material. During this internal deformation the grains are jammed and the force chains are rearranged into a more stable configuration. Although creep continues the newly formed granular package is stronger than the previous package and therefore a short period of quiescence without slip events occurs. This rearrangement can occur several times during the late interevent phase. If the internal structure reaches a critical threshold, probably determined by the contact ratio and packing density, a runoff process starts and the system changes from creeping to dynamical slip.

Other studies have shown a similar system behaviour that is attributed to intermittent criticality (Ben-Zion et al., 2003). In contrast, to the self-organized critical system, intermittent criticality implies a cyclic evolution of the fault zone, whereas the SOC only gives a general statistic fluctuation around the critical state (i.e. failure criterion). If we apply the concept of intermittent criticality, the small precursors are the expression of small scale stress perturbations along the fault zone. Overall the stress field within the granular fault zone homogenizes by increasing rearrangement of force chains, that explains the increasing frequency of events up to a certain point. Then the system is largely homogenized and is in a critical state, very close to failure, which is comparable with the state of stress in the lithosphere (Sornette et al., 1990). This behaviour has also been observed for the temporal and spatial clustering of smaller earthquakes (Hainzl, 2003).

The behaviour of dilation during the interevent cycle is even more complex and it is difficult to assign a direct relation to micromechanical processes. The observed increase in wavelength of the small amplitude oscillations could indicate a smoothing of the internal fault surface, leading to a smoother frictional response. The discrete upward and downward steps might be artificial, or the result of sensor noise. However, the strong reproducibility over multiple cycles indicates that mechanical explanations can be valid, too. For example, internal reorganization of the granular packaging leads to discrete conformations of packaging with different densities that are characteristic for each state of the system.

\subsection{Slip Modes}

\subsubsection{Criticality of Analogue Fault}

From the determined rate-and-state parameters we can now derive the critical stiffness to evaluate how close the main series experiments are to the bifurcation from stable and unstable slip. Because normal stress is constant in each series we use a stability criteria according to J. Dieterich (2007):

$$
k_{c}=\frac{\zeta \sigma_{N}}{D_{c}}
$$

According to Heslot et al. (1994) the critical stiffness normalized by normal load $\sigma_{N}$ (in their case slider mass $M$ ) is a slowly decreasing function depending on loading velocity $v_{L}$. Accordingly, we correct for loading rate $v_{L}$ with respect to the loading rate of the SHS-tests $v_{0}=0.52 \frac{\mathrm{mm}}{\mathrm{s}}$ and the scaling factor $\alpha=10$ :

$$
k(V)=k_{c}-\alpha \ln \left(\frac{v_{L}}{v_{0}}\right)
$$

Utilizing both estimates for $\zeta=(b-a)=\{0.0084,0.0130\}$, three different estimates for $D_{c}=\{50,100,200\} \mu m$ and normalizing by normal stress the normalized 

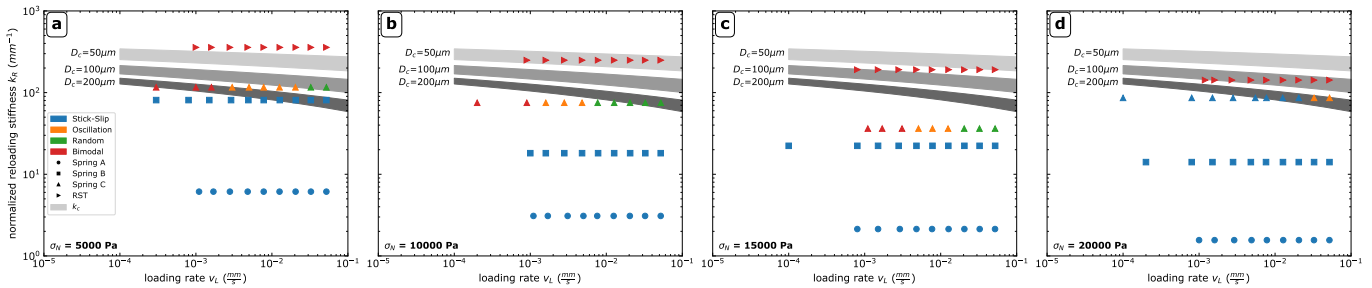

Figure 9. Slip modes in the $k \quad-\quad v$ space represented by the normalized reloading stiffness which includes the material's effect. The critical stiffness $k_{c}$ is calculated from the rate-and-state parameters from SHS-experiments at maximum stiffness (Section 3.5) and explains the transition from unstable to stable (random) slip mode for Spring C.

critical stiffness $k_{c}$ ranges between 60 and $340 \mathrm{~mm}^{-1}$. Comparing this with the normalized machine stiffness $k_{N}$ (Fig. 3) we find that most experiments show $k>k_{c}$ and therefore should only show stable sliding (J. Dieterich, 2007). However, due to the material inside the machine the actual stiffness of the system is lower. If the normalized reloading stiffness $k_{R}$ is used instead (Fig. 9), the experiments with lowest stiffness now show $k \leq k_{c}$ and the experiments with higher stiffness now show $k \approx k_{c}$.

Consequently, the experiments with Spring $\mathrm{C}(\triangle$ in Fig. 9) now fit with the change of unstable to stable slip when transitioning the stability boundary (grey boxes in Fig. 9). It is unclear why the stiffest experiments (RST) still shows unstable slip. Using a higher scaling factor $\alpha=50$ shifts the stability boundary upwards. Therefore we suspect that the scaling factor $\alpha$ might represent the ratio of normalized machine stiffness $k_{N}$ to normalized reloading stiffness $k_{R}$ which is $\frac{k_{R}}{k_{N}} \approx 50$ for RST and $\frac{k_{R}}{k_{N}} \approx 10$ for the experiments with a spring (Fig. 4). From these observations we also think that it is safe to assume that $D_{c}$ is in the order of 100 to $200 \mu \mathrm{m}$ which is roughly the radius of a glass bead $r_{G B}=150 \ldots 200 \mu m$. Due to the uncertainties in the estimation of $k_{N}$ and the high uncertainty for $D_{c}$ the values for $k_{c}$ and the location of the stability boundary are not well constrained. The uncertainty for $D_{c}$ might result from the possibility that $D_{c}$ is not constant because the thickness of the active shear zone might change during the experiment which is a primary factor for the scaling of $D_{c}$ (Marone \& Kilgore, 1993). Furthermore $D_{c}$ shows a dependency on slip velocity which could not be studied with our setup (Hatano, 2009). Nevertheless, the fits of the SHS tests and main experiments yield results that seem valid and the stability boundary lies within the same order of magnitude.

\subsubsection{Modelling of Slow Slip Events}

For natural examples the slip mode is usually identified by the relation of seismic moment $M_{0}$ and characteristic duration $T$ (e.g. Ide et al., 2007; Gomberg et al., 2016). Regular earthquakes show a much shorter duration $\left(M_{0} \propto T^{3}\right)$ in comparison with slow earthquakes $\left(M_{0} \propto T\right)$. The scaling relation leads to a characteristic separation between events of equivalent seismic moment $M_{0}$. We observe a similar separation of slow and fast events which is most prominent for the experiments at highest stiffness. Depending on the actual seismic moment the separation in nature is between 2 and 5 orders of magnitude. In the experiments at highest stiffness we observe a separation in average slip rate of 2-3 orders of magnitude. The difference in peak slip rate is up to 5 orders of magnitude. This separation is smaller but still statistically significant for lower machine stiffness where we also find oscillating slip modes. Similarly, the frictional stress drop that can be seen as a proxy for seismic moment in our experiments, shows a separation of 2 orders of magnitude which indicates that the dynamics are different for slow and fast events. 
Several studies highlight the relationship of transient slip events promoting seismic activity (A. Kato \& Ben-Zion, 2021, and references therein). The results from our study suggest that glass beads as analogue fault gouge shows a large variety of slip modes not only at high mean stresses as previously found by others (J. H. Dieterich \& Kilgore, 1996; Cain et al., 2001; Mair et al., 2002; Cui et al., 2016). Therefore, the usage of glass beads in small scale analogue models with intermediate to high stiffness at stresses in the $\mathrm{kPa}$ range is suitable to model seismic fault behaviour. For example the glass beads can be used as fault gouge in between two elastic blocks in gel-slider type models (similar to Corbi et al., 2011) or in more complex models. If the stiffness of the model is adjusted correctly (e.g. in the range of Spring C) several slip modes are possible depending on the normal stress on the fault. The normal stress regime on the fault can then be designed by geometric orientation of the fault with respect to the loading direction. More complex fault geometries, possibly forming fault networks, then lead to transient stress on the individual faults and thereby altering slip mode. These setups can be used to study the complex interplay of fault geometry and slip on individual faults, while retaining dynamic and kinematic similarity. Due to transient stress changes individual analogue faults might change their slip mode from pure stick-slip to creep and thereby changing system dynamics and the activity of other faults in the system.

Especially the temporal distribution of slow events between the occurrence of fast events highlights the possible application of glass beads as analogue fault gouge. The increased probability of slow events towards the end of the reloading time and the high stress level is similar to the behaviour observed for large fault systems that show increased seismic activity towards the end of the seismic cycle (A. Kato \& Ben-Zion, 2021). The abrupt decrease in probability just before failure might be an expression of strong locking due to jamming by shear (Bi et al., 2011) and might favour fast slip events by driving the frictional strength above a critical threshold. In general we find that the 'precursory' phase where the fault shows signs of imminent failure starts relatively early, with the onset of creep at about $50 \%$ of the reloading time. This is similar to long preparatory phases of large earthquakes (Bouchon et al., 2013) and to recent findings by Igarashi and Kato (2021). In our case the slow events do not always act as precursors because for certain conditions they occur in a repeating pattern at high stresses (e.g. Spring C at low normal stress) and therefore are reminiscent of 'similar earthquakes' (Igarashi \& Kato, 2021). Although the stress drop magnitude slowly increases over several repeating events, they do not show a clear threshold for which the slow event grows into a large event. Consequently, they only pinpoint that the fault is close to failure but not that the fault will fail with a fast slip event after a certain type of slow event.

The slip behaviour of the granular analogue is the result of the interaction of friction with the complex network of force chains that is created in the sheared bulk material (Cates et al., 1998; Daniels \& Hayman, 2008). This micromechanical mechanism creates the macroscopic behaviour that can be described with rate-and-state friction. Our findings support the hypothesis that rate-and-state like dynamics are the expression of processes that emerge close to the criticality boundary. Similar kinematic observations can be made for a range of microphysical processes and conditions (e.g. Kabla et al., 2005; Papanikolaou et al., 2013; Scuderi et al., 2015; Hecke, 2009; Lemaître \& Caroli, 2009). Denisov et al. (2016) show that force fluctuations in granular matter, of which our experiments but also earthquakes are a part of, show scaling relations that are akin to critical phenomena. Furthermore, the size and stress distribution of slip events within granular materials at the same conditions as in our experiments is found to be universally related with slip events on multiple scales and follows similar scaling relations (Uhl et al., 2015). The underlying physical mechanisms for these observations can be quite different but yielding the same results on a macroscopic scale which is similar to the description of flows using rheological equations that purely describe the observed relation of motion and stresses while the actual deformation mechanism is different in different 
fluids. Therefore, we find that our model has widespread possibility of application within seismotectonics, engineering and hazard assessment for earthquakes and landslides.

\section{Conclusions}

We have used an annular shear apparatus to characterize the stick-slip behaviour of a granular fault zone analogue composed of glass beads. Using slide-hold-slide tests the rate-and-state properties have been qualitatively evaluated and quantified. The healing rate is found to be $b=0.0057 \pm 0.0005$. The direct effect $a$ is quantified by two approaches and found to be $a=-0.0076$ for estimates from the change of peak stress with increasing reloading velocity in SHS-tests, while the procedure by Beeler et al. (2001) with a specific $\frac{v_{L}}{t_{h}}$-ratio yields $a=-0.0030 \pm 0.0030$. In both cases the material is found to be velocity weakening with a $(b-a)=0.0131 \pm 0.0031$ or $(b-a)=0.0087 \pm 0.0029$ respectively. Due to the evolution of stress during the hold phase we find the Aging law to be slightly more appropriate for our material but the results from SHS-tests only remain inconclusive. The critical slip distance $D_{c}$ is estimated to be in the sub-mm range but can not be quantified with the presented setup. The effect of machine stiffness $k_{M}$, loading rate $v_{L}$ and normal stress $\sigma_{N}$ on the slip mode is studied. We find a large variety of slip modes ranging from pure stick-slip, oscillations to bimodal slip modes within the same experiment by only varying certain extrinsic parameters which fits well with the stability boundary derived from the rate and state parameters. Low stiffness, low loading rates and high normal stresses favour pure stick-slip with small amounts of interevent creep. Higher stiffness, especially in combination with low loading rates, leads to a bimodal distribution of fast, large events that are preceded by slow, small events. The slip events reproduce typical characteristics that have been observed in similar experiments in other experimental setups with different boundary conditions and materials allowing to generalize the observations to natural occurrences of earthquakes. In the experiments, rearrangement in the granular package is the major micromechanical process which distributes and dissipates stress during shear. This drives the system closer to criticality leading to the observed precursory strengthening and the short period of quiescence before a large slip event. We conclude that the small transients can strongly affect the statistical characteristics of a single fault zone system and makes the material suitable for the use in larger analogue modelling setups that model seismotectonic deformation with a higher geometrical complexity. The small scale events during the precursory phase are the expression of distributed fluctuations of the system in a critical state. Further examination of these fluctuations and their correlation with the generation of large events may give important constraints on the predictability of slip events (as suggested by Ben-Zion et al., 2003). Furthermore, the higher complexity with differing slip modes due to the characteristics of the glass beads could provide additional insights into the system behaviour and the interaction of faults in analogue models that are closer to the behaviour of a natural fault zone. The results from this study shed light on the micromechanical mechanisms from which rate and state-friction emerges. Therefore it can act as a benchmark for numerical models of fault zones, alleviate the design of more complex analogue models and helps interpreting kinematic natural observations of fault slip.

\section{Appendix A Data Analysis}

The experimental data is examined using a combination of classical event detection, statistics and machine learning. To analyze the occurrence and properties of the slip events we employ a peak detection that is based on a minimum stress drop threshold for each experiment. Then we extract certain characteristic points in the stress curve, these are highlighted in Fig. 2c. To avoid confusion with other terms which might describe similar features Tab. A1 lists all the characteristics we use and highlights publications where a more throughout definition is found. 


\section{A1 Picking and First Order Properties}

For first order characterization of the experiments we use a simple peak detection to find slip events in the stress curve. For this the data is split into sets of equal loading rate, normal stress and stiffness. A fixed threshold for stress drop per set facilitates the detection of sudden changes in shear stress. Fine tuning this value enables the detection of large and fast, but also of small and slow events by searching for positive and negative peaks in the data. The result of peak detection is cross checked by manual inspection of the stress curves and the detected peaks (Fig. 2a+b).

The point $X$ of maximum stress immediately before failure is denoted by $X_{p}$ indicating peak values, the point of minimal stress after a slip event is indicated by $X_{e}$ accordingly (Fig. 2c). In most cases $X$ is replaced by the appropriate physical quantity such as shear stress $\tau$ or velocity $v$. A velocity threshold defines the separation of nondynamic and dynamic slip events. A slip event is considered dynamic when at any point during a decrease of shear stress the slip velocity $v_{s}$ is higher than the threshold $v_{d}$. In this study we used the maximum loading velocity of $v_{L}=0.02 \frac{\mathrm{mm}}{\mathrm{s}}$ as the threshold. This allows the definition of onset of dynamic slip, denoted by $X_{d}^{s}$, maximum slip $X_{m}$ where slip velocity is at its maximum and the end of dynamic slip $X_{f}$ where the slip velocity drops below the critical value. These points now define a full cycle, which we see as an analogue of a seismic cycle.

The full cycle is defined as the period of time between two slip events that have a dynamic phase with velocities above the threshold $v_{d}$. During the majority of a full cycle the shear stress increases in a linear relation with load point displacement but deviates to a non-linear relation. This point is the onset of creep and is defined as the point where the linear trend extrapolated from the previous points deviates by more than $1 \%$. The slope of the linear trend, calculated by least squares fitting, also defines the cyclic reloading stiffness $k_{L}$ which is a measure for the overall stiffness of the setup including the bulk stiffness of the granular material. This stiffness is also used for further calculations of the criticality using the rate-and-state framework.

Assuming an overall elastic behaviour of the granular material when completely locked, we can estimate the amount of creep either as overall proportion or as instantaneous creep. Overall creep is calculated by linearly extrapolating the shear stress increase over the full cycle using $k_{L}$ as a slope and then relating the predicted and observed point of failure. Similarly the instantaneous creep is calculated by a similar method but doing a point wise calculation.

\section{A2 The RSD-Formulation}

Following J. Dieterich (2007, and references therein), shear stress $\tau$ evolves as a function of effective normal stress $\sigma$, load point velocity $v_{L}$ and a set of experimentally derived parameters $\mu_{0}, a, b, \theta, D_{c}$ in relation to a reference load point velocity $v_{L}^{*}$ :

$$
\tau=\sigma\left[\mu_{0}+a \ln \left(\frac{v_{L}}{v_{L}^{*}}\right)+b\left(\frac{\theta v_{L}^{*}}{D_{c}}\right)\right]
$$

This is a heuristic description of the change in shear stress in response to a change in slip velocity. The parameters in Eq. A1 are usually derived experimentally using velocity stepping tests where the system sliding at a given reference load point velocity $v_{L}^{*}$ under stable conditions $(\dot{\theta}=0)$ is perturbed by setting a new loading velocity $v_{L}$. This prompts an direct reaction of shear stress that is proportional to the magnitude of the perturbation $\frac{v_{L}}{v_{L}^{*}}$ and the constant $a$ ('direct effect'). Following this immediate reaction, shear stress adjusts to a new level defined by the evolution of state over time in relation to the new loading velocity normalized by the characteristic slip distance $\frac{\theta v_{L}^{*}}{D_{c}}$ and a con- 
stant $b$ ('evolution effect'). The evolution of state over time $\dot{\theta}$ is defined by choosing one of the following evolution laws:

$$
\begin{gathered}
\dot{\theta}=1-\frac{v_{L} \theta}{D_{c}} \text { Aging Law } \\
\dot{\theta}=-\frac{v_{L} \theta}{D_{c}} \ln \frac{v_{L} \theta}{D_{c}} \text { Slip Law } \\
\dot{\theta}=e^{\frac{v_{L}}{\delta_{c}}}-\frac{v_{L} \theta}{D_{c}} \ln \frac{v_{L} \theta}{D_{c}} \text { Kato Law } \\
\dot{\theta}=1-\frac{v_{L} \theta}{D_{c}}-\frac{c}{b} \theta \frac{\dot{\tau}}{\sigma} \text { Nagata Law }
\end{gathered}
$$

The Aging law (Dieterich, 1978) and Slip law (Ruina, 1983) are the most commonly used state equations, while the Kato law (N. Kato \& Tullis, 2001) and Nagata law (Nagata et al., 2012) are more recent developments. All of the above laws contain a slip dependent component which is expressed in the term $\frac{v_{L} \theta}{D_{c}}$. Consequently, the Slip law (Eq. A3) does not show any healing when there is no slip $\left(v_{L} \rightarrow 0\right)$ which can be tested through unstressed SHS tests where the sample at rest is not under stress and thus slip along grain contacts is hindered. The Aging law (Eq. A2) shows constant healing at rest due to the 1 in the equation. This purely time-dependent effect makes the Aging law fit better to experimental data (Beeler et al., 1994). However, for large velocity steps $\left(\left|\log _{10} \frac{v_{L}}{v_{L}^{*}}\right|>\right.$ 2) the Aging law shows a linear decay that is dependent on the sign and magnitude of the step which is not in accordance with experimental data. Furthermore, the Aging law does not fit well to the state evolution during a hold phase and needs non-constant $a$, $b$ and $D_{c}$ (Bhattacharya et al., 2017). The Slip law exhibits a better fit for large velocity steps and for the evolution of state during a hold. This resulted in a reformulated version of the Slip law that accounted for time-dependent healing by N. Kato and Tullis (2001) termed Kato law by Bhattacharya et al. (2017) (Eq. A4). A further improvement to the previous laws has been proposed by Nagata et al. (2012) which incorporates the relation of the 'evolution effect' $b$ and a new constant $c$, as well as the normalized stressing rate $\frac{\dot{\tau}}{\sigma}$ (Eq. A5).

\section{A21 Tests and Derived Quantities}

We performed SHS tests with stressed hold phases (Marone, 1998) to determine the direct effect $a$, rate of healing $b$ and the appropriate state law.

Due to the evolution of state during a hold, the frictional resistance $\mu_{p}$ of a granular medium increases with the natural logarithm of the hold time $t_{h}$ and gives rise to the healing rate $b$ (Bhattacharya et al., 2017):

$$
b=\frac{\delta \Delta \mu_{p}}{\delta \ln t_{h}}
$$

This increase in $\mu_{p}$ is measured with slide-hold-slide tests. During the first slide phase a steady-state value of $\mu_{s}$ is established. Then the machine is stopped for a certain time $t_{h}$, either under stress or unstressed. Then the sample is resheared and the increase of peak strength with respect to the previously established stable sliding resistance is measured as $\Delta \mu_{p}=\mu_{p}-\mu_{s}$. Relating the results to $\ln t_{h}$ a linear increase can be measured, which is the healing rate $b$. Furthermore, the loading velocity $v_{L}$ plays an important role in the magnitude of frictional resistance after loading $\mu_{p}$ but should not in- 
fluence the healing rate $b$ (Beeler et al., 2001). Experiments at increasing $v_{L}$ therefore show the same slope $b$ but increasing $\Delta \tau_{p}$.

This effect can be exploited to determine the direct effect $a$ from slide-hold-slide tests by using a specific spacing between the realized loading rates (Fig. 9 in Beeler et al., 2001). If the ratio of the loading velocities $\frac{v_{L 1}}{v_{L 2}}$ is equal to the ratio of hold times $\frac{t_{h 2}}{t_{h 1}}$ (Eq. A7) then the increase in $\Delta \mu_{p}$ is proportional to $a \cdot \ln \frac{v_{L 1}}{v_{L 2}}$. We estimated the direct effect $a$ from the average increase in $\Delta \mu_{p}$ over all realized hold times using a set of SHS-Tests that fulfil eq. A7.

$$
\frac{v_{L 1}}{v_{L 2}}=\frac{t_{h 2}}{t_{h 1}}
$$

Furthermore, the direct effect can be measured from the offset of the y-intersect of individual fits of the SHS tests at different velocities by:

$$
a=\frac{y_{0}-y_{1}}{\ln \frac{v_{0}}{v_{1}}}
$$

Other approaches to estimate $(a-b)$ were also tested with our data. Corbi et al. (2013) defines $(a-b)$ using the peak friction $\mu_{p}$ and sliding velocity $v_{L}$ (Eq. A9). For this we determine the frictional resistance $\mu_{p}$ at the peak point $\tau_{p}$ just before a dynamic failure because at the peak, there is a plateau of shear stress and therefore the current slip rate of the fault equals the loading rate $v_{S}=v_{L}$.

$$
(a-b)=\frac{\Delta \mu_{p}}{\Delta \ln v_{L}}
$$

\section{A3 Supporting Figures and Tables}


Table A1. Terminology and definition of characteristic points.

\begin{tabular}{|c|c|c|}
\hline Term & Symbol & Definition \\
\hline Stiffness & $k_{M}$ & $\begin{array}{l}\text { Theoretical stiffness of machine calculated from spring stiffness } \\
\text { and apparatus stiffness. }\end{array}$ \\
\hline Cyclic reloading stiffness & $k_{L}$ & $\begin{array}{l}\text { Measured relationship of force increase and load point dis- } \\
\text { placement during an interevent phase. This is approximately } \\
\text { the real stiffness of the apparatus with granular material. }\end{array}$ \\
\hline Unloading stiffness & $k_{U}$ & $\begin{array}{l}\text { Measured relationship of force drop and horizontal lid displace- } \\
\text { ment during a slip event. This quantity is measured using a } \\
\text { high speed camera for each of the realized stiffnesses. }\end{array}$ \\
\hline Loading velocity & $v_{L}$ & $\begin{array}{l}\text { Rotation velocity of the shear cell during an experiment. This } \\
\text { value is defined as the velocity along the median circumfer- } \\
\text { ence of the shear cell which divides the cell area into two equal } \\
\text { compartments. }\end{array}$ \\
\hline Slip velocity & $v_{S}$ & $\begin{array}{l}\text { Velocity of the lid during an event along the same circumfer- } \\
\text { ence as the loading velocity. Calculated from } F_{D} \text { and } k_{U} \text {. }\end{array}$ \\
\hline Load point displacement & $d_{L}$ & $\begin{array}{l}\text { Horizontal displacement of the shear cell along the median cir- } \\
\text { cumference by the loading velocity. Calculated by integrating } \\
v_{L} \text { over time. }\end{array}$ \\
\hline Slip displacement & $d_{S}$ & $\begin{array}{l}\text { Horizontal displacement of the lid during a slip event. Calcu- } \\
\text { lated by integrating } v_{S} \text {. }\end{array}$ \\
\hline Lid displacement & $d_{H}$ & $\begin{array}{l}\text { Vertical displacement of the lid due to internal deformation of } \\
\text { the granular medium. The zero-level is defined as the top of } \\
\text { the shear cell. }\end{array}$ \\
\hline Package density & $\rho_{P}$ & $\begin{array}{l}\text { Density of granular material during the experiment. Calcu- } \\
\text { lated from weighted mass, shear cell area and } d_{H} \text {. }\end{array}$ \\
\hline
\end{tabular}

Slip event

Abrupt reduction in shear stress along the shear zone coinciding with a counter rotation of the lid. Has a start 'Event start' and end 'Event end' defined by characteristic points on the shear curve.

Microslip

Recurrence time

Event peak

Event end

Onset of dynamic event

End of dynamic event

Preslip

Creep

Onset of Creep
Similar to a 'Slip event' but with intensity and slip velocity a few orders of magnitude lower.

$t_{r} \quad$ Time between the end $\left(t_{e}\right)$ of a 'Slip event' and the start of the next $\left(t_{p}\right)$. The time span is named interevent phase analogous to the interseismic period for earthquakes.

Maximum shear stress before a 'Slip event'.

Minimum shear stress after a 'Slip event'

Critical point where slip velocity during an event is larger than the loading rate.

Critical point where slip velocity during an event is lower than the loading rate.

$d_{p} \quad$ Slip that happens during the acceleration of a slip event between 'Event peak' and 'Onset of dynamic event'.

Ratio of 'Slip displacement' and 'Load point displacement' during the interseismic phase. Due to permanent internal deformation at very low rates, there is a deficit between displacement that is imposed on the sample and released slip during a 'Slip event'.

Position on the shear stress curve where the reloading deviates from the linear trend (defined as 'cyclic reloading stiffness $k_{L}$ ') by more than $1 \%$. 

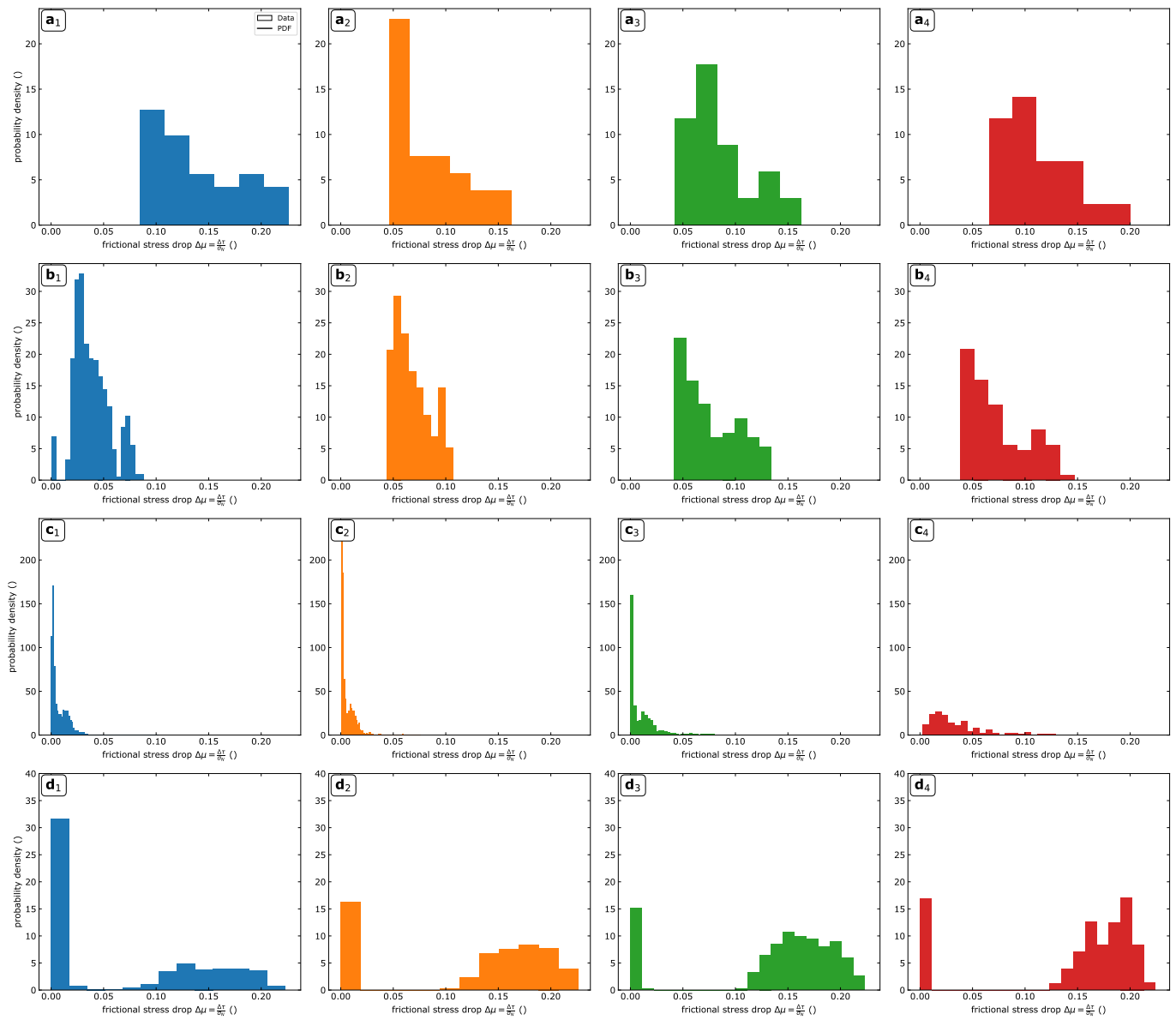

Figure A1. Histograms for frictional stress drop per experiment series. The legend in $\mathrm{a}_{1}$ applies to all plots. Each subplot summarizes data from different loading rates but at constant normal stress (indicated by colour) and constant stiffness. Each row has constant stiffness with a) $\left.k_{M}=3.3 \frac{\mathrm{kN}}{\mathrm{mm}}, \mathrm{b}\right) k_{M}=19.6 \frac{\mathrm{kN}}{\mathrm{mm}}$, c) $k_{M}=82.6 \frac{\mathrm{kN}}{\mathrm{mm}}$, and d) $k_{M}=1354.0 \frac{\mathrm{kN}}{\mathrm{mm}}$. 

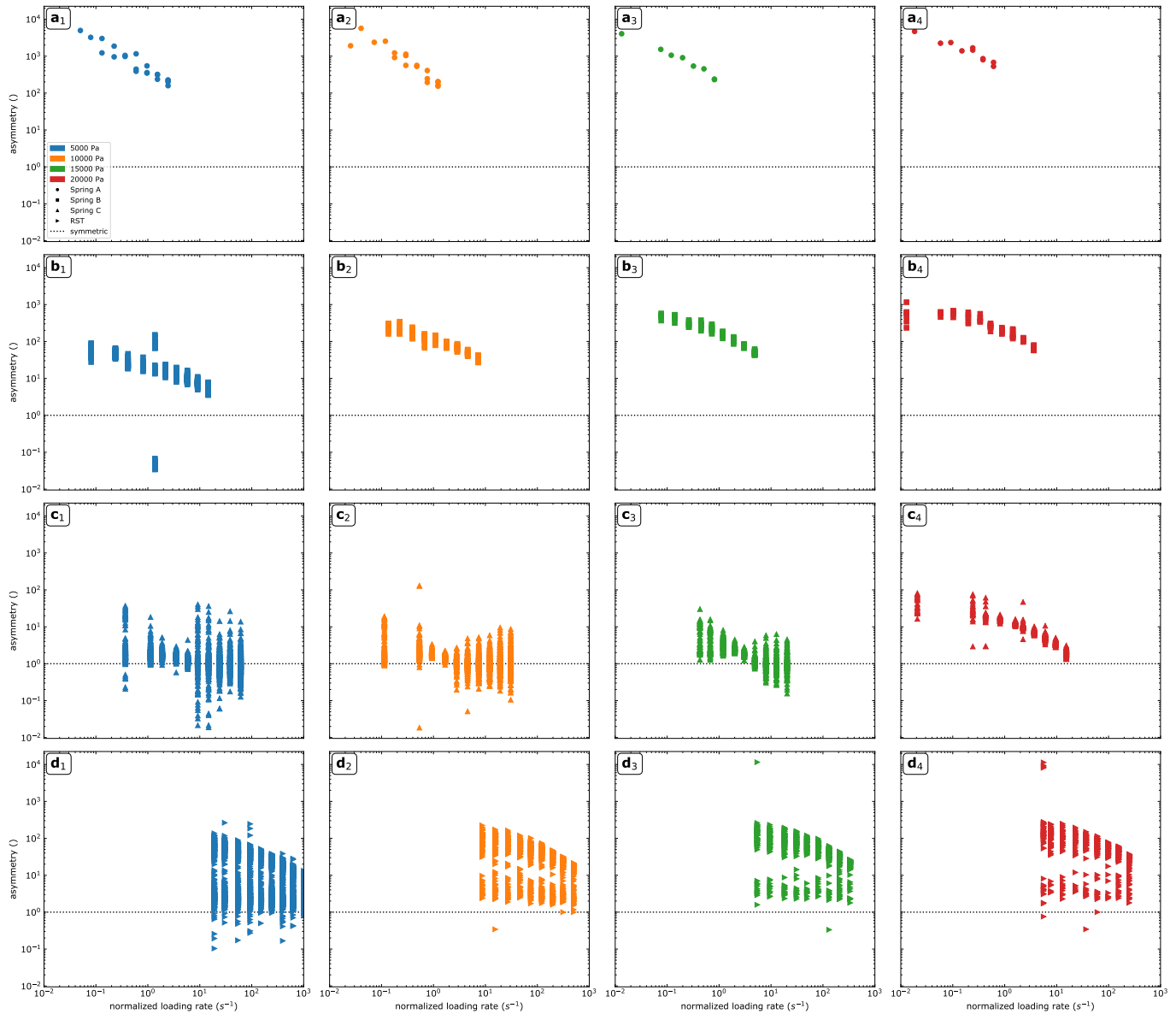

Figure A2. Asymmetry for all events and all experiments. The legend in $a_{1}$ applies for all plots. Each row represents experiments of the same stiffness which is also indicated by the individual markers. Colour highlights the different normal stresses which has an additional influence besides the stiffness and normalized loading rate. 


\section{Acknowledgments}

The work of F. Neumann and T. Ziegenhagen on the technical implementation of the setup and data acquisition system is greatly acknowledged. We thank K. Elger for the handling of the data publication. Furthermore, the authors thank the participants of the GFZ Machine Learning hackathon and colleagues from the GFZ, especially J. Münchmeyer and J. Bedford for many fruitful discussions on the dataset. Experimental data and the Python scripts used to generate the figures are available from the GFZ Data Services in the form of a data publication (Rudolf et al., in prep) https://dataservices.gfz-potsdam .de/panmetaworks/review/104236d2a3cbef19210df933fe0dec10cef2c7e965f47dd6ce3ffb533e0c57bc/. The scripts rely on the python module 'rst-stick-slipy' (Rudolf, in prep) which is going to be available after acceptance. All authors declare that no competing interests are present. This research has been funded by Deutsche Forschungsgemeinschaft (DFG) through grant number 235221301 - CRC 1114 "Scaling Cascades in Complex Systems", Project B01 "Fault networks and scaling properties of deformation accumulation".

\section{References}

Anthony, J. L., \& Marone, C. (2005). Influence of particle characteristics on granular friction. Journal of Geophysical Research: Solid Earth, 110(B8). doi: 10 $.1029 / 2004 j \mathrm{~b} 003399$

ASTM, D. (2016). Test method for bulk solids using schulze ring shear tester. doi: $10.1520 / \mathrm{d} 6773-16$

Beeler, N., Hickman, S., \& Wong, T.-f. (2001). Earthquake stress drop and laboratory-inferred interseismic strength recovery. Journal of Geophysical Research: Solid Earth, 106(B12), 30701-30713. doi: 10.1029/2000jb900242

Beeler, N., \& Tullis, T. (1997). The roles of time and displacement in velocitydependent volumetric strain of fault zones. Journal of Geophysical Research: Solid Earth, 102(B10), 22595-22609. doi: 10.1029/97jb01828

Beeler, N., Tullis, T., \& Weeks, J. (1994). The roles of time and displacement in the evolution effect in rock friction. Geophysical Research Letters, 21 (18), 19871990. doi: 10.1029/94gl01599

Ben-Zion, Y., Eneva, M., \& Liu, Y. (2003). Large earthquake cycles and intermittent criticality on heterogeneous faults due to evolving stress and seismicity. Journal of Geophysical Research: Solid Earth, 108(B6). doi: 10.1029/2002jb002121

Bhattacharya, P., Rubin, A. M., Bayart, E., Savage, H. M., \& Marone, C. (2015). Critical evaluation of state evolution laws in rate and state friction: Fitting large velocity steps in simulated fault gouge with time-, slip-, and stressdependent constitutive laws. Journal of Geophysical Research: Solid Earth, 120(9), 6365-6385. doi: 10.1002/2015jb012437

Bhattacharya, P., Rubin, A. M., \& Beeler, N. M. (2017). Does fault strengthening in laboratory rock friction experiments really depend primarily upon time and not slip? Journal of Geophysical Research: Solid Earth, 122(8), 6389-6430. doi: $10.1002 / 2017$ jb013936

Bi, D., Zhang, J., Chakraborty, B., \& Behringer, R. P. (2011). Jamming by shear. Nature, 480(7377), 355-358. doi: 10.1038/nature10667

Blank, D. G., \& Morgan, J. K. (2019). Precursory Stress Changes and Fault Dilation Lead to Fault Rupture: Insights From Discrete Element Simulations. Geophysical Research Letters, 46(6), 3180-3188. Retrieved 2021-0319, from https://agupubs.onlinelibrary.wiley.com/doi/abs/10.1029/ 2018GL081007 doi: 10.1029/2018GL081007

Bouchon, M., Durand, V., Marsan, D., Karabulut, H., \& Schmittbuhl, J. (2013, March). The long precursory phase of most large interplate earthquakes. Nature Geoscience, 6, 299. Retrieved from http://dx.doi.org/10.1038/ ngeo1770 doi: 10.1038/ngeo1770 
Brace, W., \& Byerlee, J. (1966). Stick-slip as a mechanism for earthquakes. Science, 153(3739), 990-992. doi: 10.1126/science.153.3739.990

Brinkman, B. A. W., LeBlanc, M. P., Uhl, J. T., Ben-Zion, Y., \& Dahmen, K. A. (2016, January). Probabilistic model of waiting times between large failures in sheared media. Physical Review E, 93(1), $013003 . \quad$ Retrieved 2021-0319, from https://link.aps.org/doi/10.1103/PhysRevE.93.013003 doi: 10.1103/PhysRevE.93.013003

Bürgmann, R. (2018). The geophysics, geology and mechanics of slow fault slip. Earth and Planetary Science Letters, 495, 112-134. doi: 10.1016/j.epsl.2018.04 .062

Cain, R. G., Page, N. W., \& Biggs, S. (2001, June). Microscopic and macroscopic aspects of stick-slip motion in granular shear. Physical Review E, 64(1), 016413. Retrieved 2021-02-10, from https://link.aps.org/doi/10.1103/ PhysRevE.64.016413 doi: 10.1103/PhysRevE.64.016413

Carpenter, B. M., Ikari, M. J., \& Marone, C. (2016). Laboratory observations of time-dependent frictional strengthening and stress relaxation in natural and synthetic fault gouges. Journal of Geophysical Research: Solid Earth, 121(2), 1183-1201. doi: 10.1002/2015jb012136

Cates, M., Wittmer, J., Bouchaud, J.-P., \& Claudin, P. (1998). Jamming, force chains, and fragile matter. Physical review letters, 81(9), 1841. doi: 10.1103/ physrevlett.81.1841

Chen, J., \& Spiers, C. J. (2016). Rate and state frictional and healing behavior of carbonate fault gouge explained using microphysical model. Journal of Geophysical Research: Solid Earth. doi: 10.1002/2016jb013470

Ciamarra, M. P., Lippiello, E., Godano, C., \& de Arcangelis, L. (2010). Unjamming dynamics: the micromechanics of a seismic fault model. Physical review letters, 104 (23), 238001. doi: 10.1103/physrevlett.104.238001

Corbi, F., Funiciello, F., Faccenna, C., Ranalli, G., \& Heuret, A. (2011, jun). Seismic variability of subduction thrust faults: Insights from laboratory models. Journal of Geophysical Research, 116(B6). doi: 10.1029/2010jb007993

Corbi, F., Funiciello, F., Moroni, M., Dinther, Y., Mai, P., Dalguer, L., \& Faccenna, C. (2013). The seismic cycle at subduction thrusts: 1 . insights from laboratory models. Journal of Geophysical Research: Solid Earth, 118(4), 1483-1501. doi: $10.1029 / 2012 \mathrm{jb} 009481$

Cui, D., Wu, W., Xiang, W., Doanh, T., Chen, Q., Wang, S., ... Wang, J. $\quad$ (2016, November). Stick-slip behaviours of dry glass beads in triaxial compression. Granular Matter, 19(1), 1. Retrieved 2021-03-19, from https://doi.org/ 10.1007/s10035-016-0682-5 doi: 10.1007/s10035-016-0682-5

Daniels, K. E., \& Hayman, N. W. (2008, nov). Force chains in seismogenic faults visualized with photoelastic granular shear experiments. J. Geophys. Res., 113(B11). Retrieved from http://dx.doi.org/10.1029/2008JB005781 doi: 10.1029/2008jb005781

Denisov, D. V., Lörincz, K. A., Uhl, J. T., Dahmen, K. A., \& Schall, P. $\quad$ (2016, February). Universality of slip avalanches in flowing granular matter. Nature Communications, 7, 10641. Retrieved from http://dx.doi.org/10.1038/ ncomms10641 doi: 10.1038/ncomms10641

Dieterich. (1978). Time-dependent friction and the mechanics of stick-slip. Pageoph, 116. doi: 10.1007/978-3-0348-7182-2_15

Dieterich, J. (2007). Applications of rate-and state-dependent friction to models of fault slip and earthquake occurrence. Treatise on Geophysics, 4, 107-129. doi: 10.1016/b978-044452748-6.00065-1

Dieterich, J. H. (1979a). Modeling of rock friction: 1. experimental results and constitutive equations. Journal of Geophysical Research: Solid Earth, 84 (B5), 2161-2168. doi: 10.1029/jb084ib05p02161 
Dieterich, J. H. (1979b). Modeling of rock friction: 2. simulation of preseismic slip. Journal of Geophysical Research: Solid Earth, 84(B5), 2169-2175. doi 10.1029/jb084ib05p02169

Dieterich, J. H. (1992). Earthquake nucleation on faults with rate-and statedependent strength. Tectonophysics, 211(1-4), 115-134. doi: 10.1016/ 0040-1951(92)90055-b

Dieterich, J. H., \& Kilgore, B. D. (1996). Imaging surface contacts: power law contact distributions and contact stresses in quartz, calcite, glass and acrylic plastic. Tectonophysics, 256(1), 219-239. doi: 10.1016/0040-1951(95)00165-4

Dorostkar, O., \& Carmeliet, J. (2018). Potential energy as metric for understanding stick-slip dynamics in sheared granular fault gouge: A coupled cfd-dem study. Rock Mechanics and Rock Engineering, 51(10), 3281-3294. doi: 10.1007/s00603-018-1457-6

Ellsworth, W. L., Matthews, M. V., Nadeau, R. M., Nishenko, S. P., Reasenberg, P. A., \& Simpson, R. W. (1999). A physically-based earthquake recurrence model for estimation of long-term earthquake probabilities. US Geological Survey Open-File Report, 99 (522), 22. doi: 10.3133/ofr99522

Ferdowsi, B., Griffa, M., Guyer, R., Johnson, P., Marone, C., \& Carmeliet, J. (2013). Microslips as precursors of large slip events in the stick-slip dynamics of sheared granular layers: A discrete element model analysis. Geophysical Research Letters, 40(16), 4194-4198. doi: 10.1002/grl.50813

Field, E. H., Arrowsmith, R. J., Biasi, G. P., Bird, P., Dawson, T. E., Felzer, K. R., ... Zeng, Y. (2014). Uniform california earthquake rupture forecast, version 3 (ucerf3) - the time-independent model. Bulletin of the Seismological Society of America, 104(3), 1122. Retrieved from +http://dx.doi.org/10.1785/ 0120130164 doi: $10.1785 / 0120130164$

Frye, K. M., \& Marone, C. (2002). The effect of particle dimensionality on granular friction in laboratory shear zones. Geophysical research letters, 29(19). doi: 10 $.1029 / 2002 \mathrm{gl} 015709$

Gomberg, J., Wech, A., Creager, K., Obara, K., \& Agnew, D. (2016). Reconsidering earthquake scaling. Geophysical Research Letters, 43(12), 6243-6251. doi: 10 $.1002 / 2016 \mathrm{gl} 069967$

Hainzl, S. (2003). Self-organization of earthquake swarms. Journal of Geodynamics, 35(1), 157 - 172. Retrieved from http://www.sciencedirect.com/ science/article/pii/S0264370702000601 doi: https://doi.org/10.1016/ S0264-3707(02)00060-1

Hainzl, S., Zöller, G., Brietzke, G. B., \& Hinzen, K.-G. ～(2013). Comparison of deterministic and stochastic earthquake simulators for fault interactions in the Lower Rhine Embayment, Germany. Geophysical Journal International, 195(1), 684-694. doi: 10.1093/gji/ggt271

Harris, R. A. (2017). Large earthquakes and creeping faults. Reviews of Geophysics, 55(1), 169-198. doi: 10.1002/2016rg000539

Hatano, T. (2009). Scaling of the critical slip distance in granular layers. Geophysical Research Letters, 36(18). Retrieved 2021-03-18, from https:// agupubs.onlinelibrary.wiley.com/doi/abs/10.1029/2009GL039665 doi: https://doi.org/10.1029/2009GL039665

Hecke, M. v. (2009, December). Jamming of soft particles: geometry, mechanics, scaling and isostaticity. Journal of Physics: Condensed Matter, 22(3), 033101. Retrieved 2021-03-19, from https://doi.org/10.1088/0953-8984/22/3/ 033101 doi: 10.1088/0953-8984/22/3/033101

Heslot, F., Baumberger, T., Perrin, B., Caroli, B., \& Caroli, C. (1994). Creep, stickslip, and dry-friction dynamics: Experiments and a heuristic model. Physical review $E$, 49(6), 4973. doi: 10.1103/physreve.49.4973

Hu, G., \& Bradley, J. (2018). A Bayesian spatial-temporal model with latent multivariate log-gamma random effects with application to earthquake magnitudes. 
Stat, 7(1), e179. doi: 10.1002/sta4.179

Ide, S., Beroza, G. C., Shelly, D. R., \& Uchide, T. (2007). A scaling law for slow earthquakes. Nature, $447(7140), 76$. doi: 10.1038/nature05780

Igarashi, T., \& Kato, A. (2021, March). Evolution of aseismic slip rate along plate boundary faults before and after megathrust earthquakes. Communications Earth \&3 Environment, 2(1), 1-7. Retrieved 2021-04-08, from https://www.nature.com/articles/s43247-021-00127-5 doi: 10.1038/s43247-021-00127-5

Jerolmack, D. J., \& Daniels, K. E. (2019, December). Viewing Earth's surface as a soft-matter landscape. Nature Reviews Physics, 1(12), 716-730. Retrieved 2021-03-19, from https: //www. nature. com/articles/s42254-019-0111-x doi: 10.1038/s42254-019-0111-x

Jiang, Y., Wang, G., Kamai, T., \& McSaveney, M. J. (2016). $\quad$ Effect of particle size and shear speed on frictional instability in sheared granular materials during large shear displacement. $\quad$ Engineering Geology, 210, 93-102. doi: 10.1016/j.enggeo.2016.06.005

Kabla, A., Debrégeas, G., Meglio, J.-M. d., \& Senden, T. J. (2005, August). Xray observation of micro-failures in granular piles approaching an avalanche. EPL (Europhysics Letters), 71(6), $932 . \quad$ Retrieved 2021-03-19, from https://iopscience.iop.org/article/10.1209/epl/i2005-10165-4/meta doi: $10.1209 / \mathrm{epl} / \mathrm{i} 2005-10165-4$

Kato, A., \& Ben-Zion, Y. (2021, January). The generation of large earthquakes. Nature Reviews Earth 83 Environment, 2(1), 26-39. Retrieved 2021-0218, from https://www.nature.com/articles/s43017-020-00108-w doi: 10.1038/s43017-020-00108-w

Kato, N., \& Tullis, T. E. (2001). A composite rate-and state-dependent law for rock friction. Geophysical research letters, 28(6), 1103-1106. doi: 10.1029/ $2000 \mathrm{gl} 012060$

Kawamura, H., Hatano, T., Kato, N., Biswas, S., \& Chakrabarti, B. K. (2012). Statistical physics of fracture, friction, and earthquakes. Reviews of Modern Physics, 84 (2), 839. doi: 10.1103/revmodphys.84.839

Klinkmüller, M., Schreurs, G., Rosenau, M., \& Kemnitz, H. (2016). Properties of granular analogue model materials: A community wide survey. Tectonophysics, 684, 23-38. doi: 10.1016/j.tecto.2016.01.017

Lapusta, N., \& Rice, J. R. (2003). Nucleation and early seismic propagation of small and large events in a crustal earthquake model. Journal of Geophysical Research: Solid Earth, 108(B4). Retrieved 2021-03-19, from https://agupubs .onlinelibrary.wiley.com/doi/abs/10.1029/2001JB000793 doi: https:// doi.org/10.1029/2001JB000793

Lebigot, E. O. (2021). Uncertainties: a python package for calculations with uncertainties. https://github.com/lebigot/uncertainties/. GitHub.

Leeman, J., Saffer, D., Scuderi, M., \& Marone, C. (2016). Laboratory observations of slow earthquakes and the spectrum of tectonic fault slip modes. Nature communications, 7. doi: 10.1038/ncomms11104

Lemaitre, A., \& Caroli, C. (2009). Rate-dependent avalanche size in athermally sheared amorphous solids. Physical review letters, 103(6), 065501. doi: 10 .1103 /physrevlett.103.065501

Lohrmann, J., Kukowski, N., Adam, J., \& Oncken, O. $\quad$ (2003). The impact of analogue material properties on the geometry, kinematics, and dynamics of convergent sand wedges. Journal of Structural Geology, 25(10), 1691-1711. doi: $10.1016 / \mathrm{s} 0191-8141(03) 00005-1$

Mair, K., Frye, K. M., \& Marone, C. (2002). Influence of grain characteristics on the friction of granular shear zones. Journal of Geophysical Research: Solid Earth, 107(B10). doi: 10.1029/2001jb000516 
Marone, C. (1998). Laboratory-derived friction laws and their application to seismic faulting. Annual Review of Earth and Planetary Sciences, 26 (1), 643-696. doi: 10.1146/annurev.earth.26.1.643

Marone, C., \& Kilgore, B. (1993, April). Scaling of the critical slip distance for seismic faulting with shear strain in fault zones. Nature, 362(6421), 618-621. Retrieved 2021-03-18, from https://www.nature.com/articles/362618a0 doi: $10.1038 / 362618 \mathrm{a} 0$

Marone, C., Raleigh, C. B., \& Scholz, C. (1990). Frictional behavior and constitutive modeling of simulated fault gouge. Journal of Geophysical Research: Solid Earth, 95(B5), 7007-7025. doi: 10.1029/jb095ib05p07007

Marone, C., \& Saffer, D. M. (2015, January). $\quad 4.05$ - The Mechanics of Frictional Healing and Slip Instability During the Seismic Cycle. In G. Schubert (Ed.), (pp. 111-138). Oxford: Elsevier. Retrieved 2021-03-19, from https://www .sciencedirect.com/science/article/pii/B9780444538024000920 doi: 10 .1016/B978-0-444-53802-4.00092-0

Nagata, K., Nakatani, M., \& Yoshida, S. (2012). A revised rate-and state-dependent friction law obtained by constraining constitutive and evolution laws separately with laboratory data. Journal of Geophysical Research: Solid Earth, 117(B2). doi: $10.1029 / 2011$ jb008818

Nasuno, S., Kudrolli, A., Bak, A., \& Gollub, J. P. (1998). Time-resolved studies of stick-slip friction in sheared granular layers. Physical Review E, 58(2), 2161. doi: 10.1103 /physreve. 58.2161

Nasuno, S., Kudrolli, A., \& Gollub, J. P. (1997). Friction in granular layers: Hysteresis and precursors. Physical Review Letters, 79(5), $949 . \quad$ doi: 10.1103/ physrevlett.79.949

Obara, K., \& Kato, A. (2016). Connecting slow earthquakes to huge earthquakes. Science, 353(6296), 253-257. doi: 10.1126/science.aaf1512

Papanikolaou, S., Dimiduk, D. M., Choi, W., Sethna, J. P., Uchic, M. D., Woodward, C. F., \& Zapperi, S. (2013). Quasi-periodic events in crystal plasticity and the self-organized avalanche oscillator. Nature, 517-521. doi: $10.1038 /$ nature 11568

Parsons, T. (2005). Significance of stress transfer in time-dependent earthquake probability calculations. Journal of Geophysical Research: Solid Earth, 110(B5). Retrieved 2021-03-19, from https://agupubs.onlinelibrary .wiley.com/doi/abs/10.1029/2004JB003190 doi: https://doi.org/10.1029/ 2004JB003190

Peng, Z., \& Gomberg, J. (2010, September). An integrated perspective of the continuum between earthquakes and slow-slip phenomena. Nature Geoscience, 3(9), 599-607. Retrieved 2021-04-08, from https://www.nature.com/articles/ ngeo940 doi: $10.1038 /$ ngeo940

Ritter, M. C., Leever, K., Rosenau, M., \& Oncken, O. ～(2016a). Scaling the sand box - mechanical (dis-) similarities of granular materials and brittle rock. $J$. Geophys. Res. Solid Earth. Retrieved from http://dx.doi.org/10.1002/ 2016JB012915 doi: 10.1002/2016jb012915

Ritter, M. C., Leever, K., Rosenau, M., \& Oncken, O. (2016b). Supplement to: Scaling the sand box - mechanical (dis-) similarities of granular materials and brittle rock. GFZ Data Services. Retrieved 2017-04-04, from http://doi.org/ 10.5880/GFZ.4.1.2016.005 doi: 10.5880/GFZ.4.1.2016.005

Rosenau, M., Corbi, F., \& Dominguez, S. (2017). Analogue earthquakes and seismic cycles: experimental modelling across timescales. Solid Earth, 8(3), 597. doi: $10.5194 /$ se-8-597-2017

Rosenau, M., \& Oncken, O. (2009). Fore-arc deformation controls frequency-size distribution of megathrust earthquakes in subduction zones. Journal of Geophysical Research: Solid Earth (1978-2012), 114(B10). doi: 10.1029/2009jb006359 
Rudolf, M. (in prep). Rst-stick-slipy. GitLab Repository, Helmholtz Centre Potsdam - Deutsches GeoForschungsZentrum GFZ. GitLab-Repository. Retrieved from https://git.gfz-potsdam.de/analab-code/rst-stick -slipy (notpublicyet)

Rudolf, M., Rosenau, M., \& Oncken, O. ～(in prep). Ring shear and slide-hold-slide test measurements for soda-lime glassbeads of 300$400 \mu \mathrm{m}$ diameter used at the helmholtz laboratory for tectonic modelling, potsdam, germany. GFZ Data Services. Getrieved from https://dataservices.gfz-potsdam.de/panmetaworks/review/ 104236d2a3cbef19210df933fe0dec10cef 2c7e965f47dd6ce3ffb533e0c57bc/

Ruina, A. (1983). Slip instability and state variable friction laws. Journal of Geophysical Research: Solid Earth, 88(B12), 10359-10370. doi: $10.1029 /$ jb088ib12p10359

Scholz, C. H. (1998). Earthquakes and friction laws. Nature, 391(6662), 37-42. doi: $10.1038 / 34097$

Schulze, D. (1994). Development and application of a novel ring shear tester. Aufbereitungs Technik, 35(10), 524-535.

Scuderi, M. M., Carpenter, B. M., Johnson, P. A., \& Marone, C. (2015). Poromechanics of stick-slip frictional sliding and strength recovery on tectonic faults. Journal of Geophysical Research: Solid Earth, 120(10), 6895-6912. doi: 10.1002/2015jb011983

Sornette, D., Davy, P., \& Sornette, A. (1990). Structuration of the lithosphere in plate tectonics as a self-organized critical phenomenon. Journal of Geophysical Research: Solid Earth, 95(B11), 17353-17361. Retrieved from https:// agupubs . onlinelibrary . wiley.com/doi/abs/10.1029/JB095iB11p17353 doi: 10.1029/JB095iB11p17353

Tullis, T. E., \& Weeks, J. D. (1986). Constitutive behavior and stability of frictional sliding of granite. In Friction and faulting (pp. 383-414). Springer. doi: 10 .1007/978-3-0348-6601-9_2

Uhl, J. T., Pathak, S., Schorlemmer, D., Liu, X., Swindeman, R., Brinkman, B. A. W., ... Dahmen, K. A. (2015, November). Universal quake statistics: From compressed nanocrystals to earthquakes. Scientific Reports, 5,16493. Retrieved from http://dx.doi.org/10.1038/srep16493 doi: $10.1038 /$ srep16493

Van den Ende, M., Chen, J., Ampuero, J.-P., \& Niemeijer, A. ～(2018). A comparison between rate-and-state friction and microphysical models, based on numerical simulations of fault slip. Tectonophysics, 733, 273-295. doi: $10.1016 /$ j.tecto.2017.11.040

Zöller, G., \& Hainzl, S. (2007). Recurrence time distributions of large earthquakes in a stochastic model for coupled fault systems: The role of fault interaction. Bulletin of the Seismological Society of America, 97, 1679-1687. doi: $10.1785 / 0120060262$

Zöller, G., Hainzl, S., Ben-Zion, Y., \& Holschneider, M. (2011). Seismicity, Critical States of: From Models to Practical Seismic Hazard Estimates Space. In R. A. Meyers (Ed.), (pp. 805-824). New York, NY: Springer. Retrieved 202103-19, from https://doi.org/10.1007/978-1-4419-7695-6_43 doi: 10.1007/ 978-1-4419-7695-6_43 